\title{
Topological anomalies for Seifert 3-manifolds
}

\author{
Camillo Imbimbo ${ }^{a, b}$ and Dario Rosa ${ }^{c, d, e}$ \\ ${ }^{a}$ Dipartimento di Fisica, Università di Genova, \\ Via Dodecaneso 33, 16146 Genova, Italy \\ ${ }^{b}$ INFN - Sezione di Genova, \\ Via Dodecaneso 33, 16146, Genova, Italy \\ ${ }^{c}$ School of Physics and Astronomy and \\ Center for Theoretical Physics Seoul National University, \\ Seoul 151-747, Korea \\ ${ }^{d}$ Dipartimento di Fisica, Università di Milano-Bicocca, \\ I-20126 Milano, Italy \\ ${ }^{e}$ INFN - Sezione di Milano-Bicocca, \\ I-20126 Milano, Italy \\ E-mail: camillo.imbimbo@ge.infn.it, dario.rosa85@snu.ac.kr
}

ABSTRACT: We study globally supersymmetric 3d gauge theories on curved manifolds by describing the coupling of $3 \mathrm{~d}$ topological gauge theories, with both Yang-Mills and Chern-Simons terms in the action, to background topological gravity. In our approach, the Seifert condition for manifolds supporting global supersymmetry is elegantly deduced from the BRST transformations of topological gravity. A cohomological characterization of the geometrical moduli which affect the partition function is obtained. In the Seifert context the Chern-Simons topological (framing) anomaly is BRST trivial. We compute explicitly the corresponding local Wess-Zumino functional. As an application, we obtain the dependence on the Seifert moduli of the partition function of 3d supersymmetric gauge theory on the squashed sphere by solving the anomalous topological Ward identities, in a regularization independent way and without the need of evaluating any functional determinant.

KEYwords: Topological Field Theories, BRST Symmetry, Anomalies in Field and String Theories, Supersymmetric gauge theory

ARXIV EPRINT: 1411.6635 


\section{Contents}

1 Introduction $\quad 1$

2 Coupling 3d Chern-Simons to topological gravity 4

3 Coupling 3d topological YM with CS term to topological gravity $\quad 8$

4 The supersymmetric point $\quad 11$

5 The relation between topological and physical supersymmetry 12

6 Topological Anomaly for Seifert manifolds 17

$\begin{array}{llr}7 & \text { Moduli } & 20\end{array}$

8 The topological anomaly for the squashed spheres $\quad 24$

9 Conclusions $\quad 26$

$\begin{array}{ll}\text { A Squashed 3-spheres in the Hopf coordinates } & 28\end{array}$

B Gravitational CS action and the Seifert WZ action 29

$\begin{array}{ll}\text { C Chern-Simons supergravity actions } & 30\end{array}$

\section{Introduction}

In the last few years there has been considerable progress in the analytical evaluation of partition functions and observables of supersymmetric gauge theories in different dimensions on certain compact manifolds equipped with suitable metrics. The common theme of these computations is localization. Localization is a long-known property of supersymmetric and topological theories, by virtue of which semi-classical approximation becomes, in certain cases, exact [1-3]. In more recent times this property has been exploited with considerable success in the work by Pestun [4] and in many following papers. In Pestun's approach no twisting of supersymmetry is performed. One rather seeks for manifolds and metrics supporting (generalized) covariantly constant spinors which ensure that certain supersymmetry global charges are unbroken. The global supersymmetry charges, even if spinorial in character, function essentially as topological BRST charges. Under favourable conditions one can choose a Lagrangian for which the semi-classical computation in the supersymmetric background is exact. 
In three dimensions, a host of results is available. Explicit exact computations have been performed for 3-spheres, both with round and "squashed" metrics, and for Lens spaces. The best understood case is the one for which the complex conjugate of the (generalized) covariantly constant spinor is also covariantly constant. This is referred to as the "real" case in [5]. In all these cases the existence of (generalized) convariantly constant spinors implies in turn the existence of a Seifert structure on the 3-manifold. This refers to 3-manifolds with an almost contact metric structure and associated Reeb Killing vector field.

As a matter of fact Seifert 3-manifolds had already made their appearance earlier, in the study of non-supersymmetric pure Chern-Simons (CS) gauge theories. It was discovered first "experimentally" [6,7] and then explained using various approaches by different authors $[8,9]$ that the semiclassical approximation for CS theories becomes exact precisely for Seifert 3-manifolds. Later, starting from [10], this result was rederived by considering the supersymmetric extension of CS: indeed this model is equivalent, after integrating out non-dynamical auxiliary fields, to the bosonic theory. In this way, computability of CS on Seifert manifolds was brought within the more general paradigm of convariantly constant spinors and localization.

In some cases, it is possible to perform localization computations not just for a single isolated Seifert structure, but for families of Seifert metrics depending on some continuous parameters. A significant example is provided by the squashed metric on the 3 -sphere [11]. ${ }^{1}$ In those instances the partition function (and the observables) turns out to depend nontrivially on (some of) those parameters. Take for example the case of (supersymmetric) Chern-Simons theory on the squashed spheres, with the metric

$$
d s^{2}=\bar{g}_{\mu \nu}(x ; b) d x^{\mu} \otimes d x^{\nu}=\left(\sin ^{2} \theta+b^{4} \cos ^{2} \theta\right) d \theta^{2}+\cos ^{2} \theta d \phi_{1}^{2}+b^{4} \sin ^{2} \theta d \phi_{2}^{2} .
$$

At first sight, the fact that the partition function is a non-trivial function of the squashing parameter $b^{2}$ is not, per se, surprising. Indeed, even if CS theory is topological at classical level, topological invariance is anomalous at quantum level [13]. This means that the quantum CS action does depend on the background metric in a way which is controlled by the anomaly functional:

$$
\delta \Gamma_{\mathrm{CS}}\left[g_{\mu \nu}\right]=\frac{c}{6} \int_{M_{3}} A_{1}^{(3)}\left[g_{\mu \nu}, \psi_{\mu \nu}\right]=\frac{c}{6} \int_{M_{3}} \epsilon^{\mu \nu \rho} R_{\mu}^{\alpha} D_{\nu} \psi_{\rho \alpha} d^{3} x,
$$

where $\psi_{\mu \nu}=\delta g_{\mu \nu}$ is the variation of the metric and $c$ is a computable anomaly coefficient. However, if one plugs both the squashed metric (1.1) and its variation $\psi_{\mu \nu}=b \partial_{b} g_{\mu \nu}$ into the anomaly form (1.2), one finds that the anomaly vanishes identically

$$
A_{1}^{(3)}\left[g_{\mu \nu}(x ; b), b \partial_{b} g_{\mu \nu}\right]=0 \text {. }
$$

In this work we will solve this conundrum: we will see that the vanishing of the topological anomaly for the squashed spheres is compatible with the non-trivial dependence of the

\footnotetext{
${ }^{1}$ In this paper, we will consider the squashing of the 3 -sphere that in [11] is referred to as the "less familiar" one. This is the squashing that preserves only the $\mathrm{U}(1) \times \mathrm{U}(1)$ isometry. A related deformation of the round sphere, the branched sphere $S_{q}^{3}$, is discussed in [12].
} 
partition function on the squashing parameter. As a matter of fact, we will show that the topological anomaly captures the precise dependence of the partition function on $b$. We will extend this results to generic three-dimensional supersymmetric theories, with both YangMills and Chern-Simons terms in the action (YM+CS), involving vector multiplets only.

The resolution of our puzzle will require understanding the appropriate renormalization prescription for quantum effective actions on Seifert manifolds. The time-honored method to identify the renormalization prescriptions associated to certain symmetries is to introduce backgrounds fields which act as sources for the currents associated to those symmetries. This approach has been forcefully advocated more recently in the specific context of supersymmetric gauge theories in [14] and in several following papers.

We also will introduce backgrounds, but our treatment will differ from the one which has become common in the literature on localization of the last few years. Instead of coupling the supersymmetric gauge theory to supergravity, we will first consider its topological version and then couple it to topological gravity.

This will have the advantage of obtaining the Seifert condition for global supersymmetry in the most straightforward way by avoiding all the complications of spinors. Moreover and most importantly the topological gravity formulation will make immediate to identify the subsets of the topological transformations which preserve the Seifert structure. These topological Seifert BRST transformations lead to a cohomological characterization of the geometrical moduli which affect the partition function. We compute the cohomology which parametrizes Seifert moduli in section 7.

In the Seifert context the topological (also known as "framing") anomaly is BRST trivial [8]. In this paper we compute explicitly the corresponding local Wess-Zumino (WZ) functional. To our knowledge, this has not been done before. We will call this functional the Seifert WZ action and write it down in eq. (6.23). The Seifert WZ action is a local functional which is invariant under Seifert-preserving reparametrizations and whose BRST variation equals the topological anomaly for Seifert manifolds. We will use the Seifert WZ action to derive the dependence on the Seifert moduli of the quantum action directly from the anomalous Ward identity associated to the topological anomaly. Our computation will be manifestly regularization and gauge independent: we will do it without the need of computing explicitly any functional determinant.

Let us clarify the relationship between our Seifert WZ action and the CS gravitational functional. It is well known that the topological anomaly (1.2) can be expressed, for generic (i.e. also non-Seifert) 3-manifolds, as a BRST variation

$$
A_{1}^{(3)}=s \Gamma_{\mathrm{GCS}}^{(3)}[g]
$$

where $\Gamma_{\mathrm{GCS}}^{(3)}[g]$ is the 3-dimensional gravitational Chern-Simons action

$$
\Gamma_{\mathrm{GCS}}^{(3)}[g]=\operatorname{Tr}\left(\frac{1}{2} \Gamma d \Gamma+\frac{1}{3} \Gamma^{3}\right)
$$

$\Gamma_{\mathrm{GCS}}^{(3)}[g]$ is not a globally defined 3-form and thus it is not a legitimate Wess-Zumino local action. This is why $A_{1}^{(3)}$ is a genuine anomaly (for generic 3 -manifolds). It is possibile how- 
ever to trade full ${ }^{2}$ reparametrization invariance with topological invariance by considering the renormalization prescription in which one subtracts the gravitational CS action from the non-local quantum action $\Gamma_{\mathrm{CS}}[g]$. The resulting renormalized action

$$
\tilde{\Gamma}_{\mathrm{CS}}[g]=\Gamma_{\mathrm{CS}}[g]-\Gamma_{\mathrm{GCS}}^{(3)}[g]
$$

is topological (i.e. it is independent of the metric) but it is not fully reparametrization invariant: it depends on the framing. The fact that the topological anomaly, when evaluated for the squashed sphere background, vanishes (eq. (1.3)), means that the gravitational Chern-Simons action, when evaluated on the same background, is a constant independent of $b$. Hence eq. (1.6) implies that also the non-local quantum action $\Gamma_{\mathrm{CS}}[g]$ evaluated for the squashed sphere is $b$-independent. In other words the gravitational CS action does not capture the dependence of the partition function on the squashing parameter. As we will explain in detail in section 6 , the reason for this is that the gravitational CS action is not the counterterm appropriate for renormalizing supersymmetric theories on Seifert manifolds. The correct counterterm is the one which respects the relevant - reparametrization and topological - Seifert simmetries: and it is precisely our Seifert Wess-Zumino action. In appendix B we show that the gravitational CS action and the Seifert Wess-Zumino action differ by a total derivative which does not integrate to zero on Seifert manifolds.

In the supergravity context, the gravitational CS action (1.5) admits a supersymmetric extension which has been discussed in $[15,16]$. We will show in appendix $\mathrm{C}$ that also this supergravity CS local action does not capture the correct dependence on the Seifert moduli, for the same reason mentioned in the paragraph above: the counterterm which respects the Seifert symmetry is a supersymmetrization of our Seifert WZ action (6.23) which involves both gravitational and gauge fields. The correct $b$-dependence of the quantum action is obtained by taking the difference of the supergravity CS action evaluated for the squashed background and the supersymmetric Seifert WZ action. In the same appendix we also show that the gauge counterterm which supersymmetrize the Seifert WZ action (6.23) does not contribute to the overall moduli dependence of the partition function. The full moduli dependence is still captured by the same Seifert WZ action which emerges in the topological gravity framework. In this way we verify directly that both the topological and the supergravity language lead to the same answer.

\section{Coupling 3d Chern-Simons to topological gravity}

The classical Chern-Simons action $[17-21]$ is

$$
\Gamma_{\mathrm{CS}}=\int_{M_{3}} \operatorname{Tr}\left[\frac{1}{2} A d A+\frac{1}{3} A^{3}\right]
$$

where

$$
A=A_{\mu}^{a} T^{a} d x^{\mu}
$$

\footnotetext{
${ }^{2}$ By full reparametrization invariance we mean invariance under both "small" and "large" coordinate transformations.
} 
is a 1 -form gauge field on a closed 3 -manifold $M_{3} . T^{a}$, with $a=1, \ldots, \operatorname{dim} G$, are generators of the Lie algebra of the simple, connected and simply connected gauge group $G$. Gauge invariance leads to the nilpotent BRST transformation rules ${ }^{3}$

$$
S_{0} A=-D c, \quad S_{0} c=-c^{2},
$$

where $c=c^{a} T^{a}$ is the ghost field carrying ghost number +1 and $D c \equiv d c+[A, c]_{+}$is the covariant differential.

The classical action (2.1) is both invariant under diffeomorphisms and independent of the 3-dimensional background metric $g_{\mu \nu}$. In order to study the fate in the quantum theory of this global topological symmetry one must couple the theory to suitable backgrounds. This has been done in [22] where it is explained that the backgrounds appropriate for the topological symmetry in question are those of equivariant topological gravity [23-26]

$$
\begin{aligned}
s g_{\mu \nu} & =\psi_{\mu \nu}-\mathcal{L}_{\xi} g_{\mu \nu}, & s \psi_{\mu \nu} & =\mathcal{L}_{\gamma} g_{\mu \nu}-\mathcal{L}_{\xi} \psi_{\mu \nu}, \\
s \xi^{\mu} & =\gamma^{\mu}-\frac{1}{2} \mathcal{L}_{\xi} \xi^{\mu}, & s \gamma^{\mu} & =-\mathcal{L}_{\xi} \gamma^{\mu},
\end{aligned}
$$

where $\xi^{\mu}$ is the ghost of reparametrizations of ghost number $+1, \psi_{\mu \nu}$ is the topological gravitino of ghost number +1 and $\gamma^{\mu}$ is the ghost-for-ghost of ghost number +2 .

The coupling to background topological gravity induces both deformations in the BRST transformations of the matter fields and extra terms in the action. It also requires introducing new matter fields $\tilde{A}$ and $\tilde{c}$ which sit in the same BRST multiplet as $c$ and $A$. $\tilde{A}$ and $\tilde{c}$ are Lie algebra-valued 2 and 3 -forms

$$
\tilde{A}=(\tilde{A})_{\mu \nu}^{a} T^{a} d x^{\mu} d x^{\nu}, \quad \tilde{c}=(\tilde{c})_{\mu \nu \rho}^{a} T^{a} d x^{\mu} d x^{\nu} d x^{\rho},
$$

of ghost number -1 and -2 respectively. All the matter fields fit niceley into a single super-field, or polyform, $\mathcal{A}$,

$$
\mathcal{A} \equiv c+A+\tilde{A}+\tilde{c}
$$

whose total fermionic number, given by the form degree plus ghost number, is $+1 .^{4}$ The action of the BRST transformation on the supermultiplet (2.6) before coupling to topological gravity writes in the compact form

$$
\delta_{0} \mathcal{A}+\mathcal{A}^{2}=0
$$

where

$$
\delta_{0}=S_{0}+d, \quad \delta_{0}^{2}=0
$$

is the "rigid" coboundary operator of total fermion number +1 . It is immediate to check that (2.7) reduces to (2.4) when restricted to the fields $c$ and $A$.

\footnotetext{
${ }^{3}$ We will adopt the convention that BRST operator $S_{0}$ anti-commutes with the exterior differential $d$.

${ }^{4}$ The benefit of polyforms for analyzing the BRST structure of flat Chern-Simons theory was first pointed out in [27].
} 
To describe the coupling to topological gravity it is convenient to consider the operator

$$
S \equiv s+\mathcal{L}_{\xi},
$$

rather than the nilpotent BRST operator $s$. On the functionals of the backgrounds independent of $\xi^{\mu}, S$ satisfies

$$
S^{2}=\mathcal{L}_{\gamma}
$$

and it is therefore nilpotent on the space of equivariant functionals of the topological gravity multiplet.

After these preliminaries, one discovers that the coboundary operator appropriate to describe the BRST transformation rules of the system after coupling to topological gravity is simply

$$
\delta \equiv S+d-i_{\gamma}, \quad \delta^{2}=0,
$$

where $\delta$ writes in the same form as the rigid ones

$$
\delta \mathcal{A}+\mathcal{A}^{2}=0 .
$$

When written for the component fields, these transformations become

$$
\begin{aligned}
s c & =-c^{2}-\mathcal{L}_{\xi} c+i_{\gamma}(A), \\
s A & =-D c-\mathcal{L}_{\xi} A+i_{\gamma}(\tilde{A}), \\
s \tilde{A} & =-[\tilde{A}, c]-\mathcal{L}_{\xi} \tilde{A}-F+i_{\gamma}(\tilde{c}), \\
s \tilde{c} & =-[\tilde{c}, c]-\mathcal{L}_{\xi} \tilde{c}-D \tilde{A} .
\end{aligned}
$$

The action also gets modified after coupling to topological gravity: one can check that

$$
\Gamma_{\mathrm{CS}+\mathrm{t} . \mathrm{g} .}=\Gamma_{\mathrm{CS}}+\frac{1}{2} \int_{M_{3}} \operatorname{Tr} i_{\gamma}(\tilde{A}) \tilde{A}
$$

is invariant by transforming both the fields according to (2.13) and the backgrounds according to (2.4).

The fields $\tilde{A}$ and $\tilde{c}$ have a natural interpretation, in the Batalin-Vilkovisky formalism, as the anti-fields of $A$ and $c$. In this traditional BV framework, which is the one adopted in $[22,27]$, the action $\Gamma$ should be supplemented with the canonical piece for all the fields and backgrounds:

$$
\Gamma_{\mathrm{can}}=\int_{\Sigma} s c \tilde{c}+s A \tilde{A}+s \tilde{A} \tilde{A}+s \tilde{c} \tilde{c}+s g_{\mu \nu} \tilde{g}^{\mu \nu}+s \psi_{\mu \nu} \tilde{\psi}^{\mu \nu}+s \gamma^{\mu} \tilde{\gamma}_{\mu} .
$$

The full BV action

$$
\Gamma_{\mathrm{BV}}=\Gamma_{\mathrm{CS}+\text { t.g. }}+\Gamma_{\mathrm{can}},
$$

generates the BRST transformations of both fields and anti-fields via the familiar BV formulas. 
However in this paper we will argue that an alternative - although exotic - point of view is available in our context. In the approach that we propose $\tilde{A}$ and $\tilde{c}$ are independent auxiliary fields whose function is to close the BRST transformations off-shell: at the same time, our action we will be just $\Gamma_{\mathrm{CS}+\text { t.g. }}$, not the full $\Gamma_{\mathrm{BV}}$.

This approach is consistent since the BRST transformations close on the fields

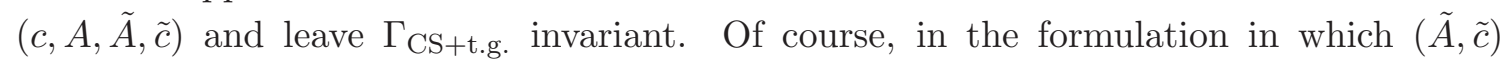
are auxiliary fields, $\Gamma_{\mathrm{CS}+\text { t.g. }}$ maintains the original non-abelian gauge symmetry, which, eventually, will have to be fixed: both the gauge non-abelian symmetry and the global vector supersymmetry associated to the topological invariance of the matter theory are captured by the BRST symmetry in (2.13). In other words, the anti-fields of the BV formulation can be reinterpreted as the auxiliary fields which are necessary to close the global supersymmetry algebra of the supersymmetric CS, after twisting that supersymmetry to obtain a topological model.

As a matter of fact, the $3 \mathrm{~d}$ action (2.14) has more local gauge invariance than just the standard non-abelian gauge invariance: it is invariant also under the fermionic local symmetry

$$
\tilde{A} \rightarrow \tilde{A}+i_{\gamma}(\chi)
$$

where $\chi$ is a fermionic scalar gauge parameter in the adjoint of the gauge group. Thus, the commuting field $\tilde{c}$ can be viewed as the ghost associated to this additional local symmetry. This extra gauge invariance is fixed by replacing $\Gamma_{\mathrm{CS}+\text { t.g. }}$ with

$$
\begin{aligned}
\Gamma_{\text {CS+t.g. }}^{\prime}= & \Gamma_{\text {CS }+ \text { t.g. }}+s \int_{M_{3}} \operatorname{Tr}\left(b * i_{\gamma}(* \tilde{A})\right) \\
= & \Gamma_{\mathrm{CS}}+\frac{1}{2} \int_{M_{3}} \operatorname{Tr} i_{\gamma}(\tilde{A}) \tilde{A}+\int_{M_{3}} \operatorname{Tr}\left[\Lambda * i_{\gamma}(* \tilde{A})-b * i_{\gamma}\left(* i_{\gamma}(\tilde{c})\right)+b * i_{\gamma}(* F)\right] \\
& +\int_{M_{3}} d^{3} x \psi_{\mu \nu} \epsilon^{\alpha \beta \mu} \gamma^{\nu} \operatorname{Tr} b \tilde{A}_{\alpha \beta},
\end{aligned}
$$

where $b$ is a 0 -form anti-ghost of ghost number -2 ,

$$
s b=-\mathcal{L}_{\xi} b-[c, b]+\Lambda, \quad s \Lambda=-\mathcal{L}_{\xi} \Lambda-[c, \Lambda]+i_{\gamma}(D b),
$$

$\Lambda$ is a Lagrange multiplier of ghost number -1 and $*$ is the Hodge dual with respect to the background metric $g_{\mu \nu}$.

Summarizing: the action $\Gamma_{\mathrm{CS}+\text { t.g. }}^{\prime}$ has the background topological supersymmetry captured by (2.4) and (2.13) and no other local gauge-invariance beyond the standard nonabelian gauge invariance: $\tilde{A}$ and $\tilde{c}$ can now be consistently thought of as auxiliary, non propagating, fields rather than anti-fields. 


\section{Coupling 3d topological YM with CS term to topological gravity}

The 3d topological YM theory is characterized by the BRST transformations

$$
\begin{aligned}
S_{0} c & =-c^{2}+\sigma, \\
S_{0} A & =-D c+\Psi, \\
S_{0} \Psi & =-[c, \Psi]-D \sigma, \\
S_{0} \sigma & =-[c, \sigma],
\end{aligned}
$$

$\Psi$ is a fermionic 1-form of ghost number 1 and $\sigma$ a bosonic 0 -form of ghost number 2. Both of them are in the adjoint of the gauge group. It is convenient to introduce a super-field or polyform of total fermionic number (ghost number + form degree) equal to 2 :

$$
\mathcal{F}=F+\Psi+\sigma .
$$

The transformations (3.1) write in a nice compact form in terms of the "rigid" coboundary operator $\delta_{0}=S_{0}+d$

$$
\mathcal{F}=\delta_{0} \mathcal{A}_{\mathrm{YM}}+\mathcal{A}_{\mathrm{YM}}^{2},
$$

where

$$
\mathcal{A}_{\mathrm{YM}}=c+A .
$$

It is important to observe that the super-field or polyform containing the gauge connection which is appropriate for the YM theory is not the same as the connection polyform $\mathcal{A}(2.6)$ of CS.

Let us again denote by $s$ the nilpotent BRST operator after coupling to topological gravity. As seen in the previous section, it is convenient to introduce the operator

$$
S_{\mathrm{YM}} \equiv s+\mathcal{L}_{\xi}, \quad S_{\mathrm{YM}}^{2}=\mathcal{L}_{\xi},
$$

where $\xi$ is the ghost associated to reparametrizations. The coboundary operator for topological YM coupled to topological gravity is again given by a formula identical to (2.11)

$$
\delta_{\mathrm{YM}} \equiv S_{\mathrm{YM}}+d-i_{\gamma}, \quad \delta_{\mathrm{YM}}^{2}=0,
$$

with $\delta_{\mathrm{YM}}$ satisfying

$$
\mathcal{F}=\delta_{\mathrm{YM}} \mathcal{A}_{\mathrm{YM}}+\mathcal{A}_{\mathrm{YM}}^{2} .
$$

These transformations write in components:

$$
\begin{aligned}
S_{\mathrm{YM}} c & =-c^{2}+i_{\gamma}(A)+\sigma, \\
S_{\mathrm{YM}} A & =-D c+\Psi, \\
S_{\mathrm{YM}} \Psi & =-[c, \Psi]+i_{\gamma}(F)-D \sigma, \\
S_{\mathrm{YM}} \sigma & =-[c, \sigma]+i_{\gamma}(\Psi) .
\end{aligned}
$$


Finally, the action of pure topological YM consists only of a $s$-trivial term:

$$
\Gamma_{\mathrm{YM}+\mathrm{t.g.}}=S_{\mathrm{YM}} \chi .
$$

As just remarked, the superfield (3.4) appropriate for YM theory is quite different from the corresponding polyform relevant for Chern-Simons theory. However, we will now show that it is possibile to recast the topological YM BRST transformations purely in terms of the Chern-Simons superfield $\mathcal{A}$. To this end, let us pick a contact structure $k$ on the 3-manifold, $M_{3}$ which is dual to the background vector field $\gamma^{\mu}$ :

$$
i_{\gamma}(k)=1, \quad \mathcal{L}_{\gamma} k=0 .
$$

1-forms $\omega$ on $M_{3}$ are naturally decomposed along the horizontal and vertical directions as follows

$$
\omega=k \omega_{V}+\omega_{H}
$$

where

$$
\omega_{V} \equiv i_{\gamma}(\omega), \quad i_{\gamma}\left(\omega_{H}\right)=0 .
$$

Let us therefore decompose the fermionic 1-form $\Psi$ of the topological YM multiplet according to

$$
\Psi \equiv k \zeta+\Psi_{H},
$$

with $i_{\gamma}(\Psi)=\zeta$. Since any horizontal form is $i_{\gamma}$-exact

$$
\Psi_{H}=i_{\gamma}(\tilde{A}),
$$

we have

$$
\Psi \equiv k \zeta+i_{\gamma}(\tilde{A}),
$$

where $\tilde{A}$ is a 2 -form of ghost number -1 . Note that the decomposition (3.15) is not unique and it has the gauge invariance

$$
\tilde{A} \rightarrow \tilde{A}+i_{\gamma}(\tilde{c})
$$

When written in terms of $\zeta$ and $\tilde{A}$ the YM topological transformations (3.8) rewrite as

$$
\begin{aligned}
S_{\mathrm{YM}} c & =-c^{2}+i_{\gamma}(A)+\sigma, \\
S_{\mathrm{YM}} A & =-D c+i_{\gamma}(\tilde{A})+k \zeta, \\
S_{\mathrm{YM}} \tilde{A} & =-[c, \tilde{A}]-F+i_{\gamma}(\tilde{c})+k D \sigma, \\
S_{\mathrm{YM}} \tilde{c} & =-[c, \tilde{c}]-D \tilde{A}-[\sigma, \tilde{A}]+k d k \zeta, \\
S_{\mathrm{YM}} \sigma & =-[c, \sigma]+\zeta, \\
S_{\mathrm{YM}} \zeta & =-[c, \zeta]+i_{\gamma}(D \sigma),
\end{aligned}
$$


where we introduced the ghost-for-ghost $\tilde{c}$ to take into account the gauge-invariance (3.16). When written in this form, the emergence of the CS superfield

$$
\mathcal{A}=\mathcal{A}_{\mathrm{YM}}+\tilde{A}+\tilde{c}
$$

becomes apparent. Indeed the transformations (3.17) can be expressed entirely in terms of $\mathcal{A}$ :

$$
\delta_{\mathrm{YM}} \mathcal{A}+\mathcal{A}^{2}=\boldsymbol{\Phi},
$$

where $\boldsymbol{\Phi}$ is the polyform of total ghost number +2

$$
\mathbf{\Phi}=\sigma+k \zeta+k D \sigma+k(d k \zeta-[\sigma, \tilde{A}]) .
$$

eq. (3.19) is perfectly equivalent to the original (3.8): by means of the decomposition associated to the contact form $k$, it was possible to reformulate the topological YM transformations in $3 \mathrm{~d}$ in terms of the full CS multiplet.

The important observation now is that it is possible to recast (3.19) in the CS-form

$$
\delta_{\mathrm{YM}} \mathcal{A}^{\prime}(\mathcal{A})+\mathcal{A}^{\prime}(\mathcal{A})^{2}=0,
$$

where

$$
\begin{aligned}
\mathcal{A}^{\prime}(\mathcal{A}) & \equiv \mathcal{A}+\boldsymbol{\Theta}, \\
\Theta & \equiv k \sigma+k d k \sigma .
\end{aligned}
$$

The relation (3.21) shows that the YM BRST operator $\delta_{\mathrm{YM}}$ has the same algebraic content as the CS BRST operator $\delta$. As a matter of fact one sees from (3.17) that $\delta_{\mathrm{YM}}$ differs from the CS $\delta$ only because it also includes, on top of the CS transformations, the shift symmetry

$$
A \rightarrow A+k \zeta
$$

together with $\sigma$, the BRST partner of $\zeta$. This shift symmetry was originally introduced in [8], to explain localization of CS theory on Seifert manifolds.

Since $\zeta, \sigma$ make a trivial BRST doublet, the physical content of $\delta_{\mathrm{YM}}$ and $\delta$ is the same. Indeed, from (3.22) one derives the identity

$$
S_{\mathrm{YM}} \Gamma\left[\mathcal{A}^{\prime}(\mathcal{A})\right]=\left.S_{\mathrm{CS}} \Gamma[\mathcal{A}]\right|_{\mathcal{A} \rightarrow \mathcal{A}^{\prime}(\mathcal{A})} .
$$

Thus, given any action $\Gamma[\mathcal{A}]$ invariant under the CS BRST operator $S_{\mathrm{CS}}$, we can obtain an action invariant under $S_{\text {YM }}$ by performing the substitutions

$$
c^{\prime}=c, \quad A^{\prime}=A+k \sigma, \quad \tilde{A}^{\prime}=\tilde{A}, \quad \tilde{c}^{\prime}=\tilde{c}+k d k \sigma .
$$

In particular from the $S_{\mathrm{CS}}$-invariant action (2.14), one obtains the $S_{\mathrm{YM}}$ invariant action

$$
\tilde{\Gamma}_{\mathrm{CS}}[A, \tilde{A}, \sigma]=\Gamma_{\mathrm{CS}}[A+k \sigma]+\frac{1}{2} \int_{M_{3}} \operatorname{Tr} i_{\gamma}(\tilde{A}) \tilde{A}
$$


which is equivalent to the CS action coupled to topological gravity backgrounds. This action is essentially the same as the Beasley-Witten's action [8]. In the symplectic formalism of [8] the term quadratic in $\tilde{A}$ is interpreted as the symplectic 2-form on the space of connections living on the base of the Seifert fibration. In our approach this term emerges naturally from the coupling to the topological backgrounds.

Summarizing, it is possible to include a CS-term in the action for the topological YM gauge theory coupled to topological gravity: this is given in (3.26). The total action of the topological YM+CS system coupled to the topological gravity backgrounds has therefore the form

$$
\Gamma_{\mathrm{YM}+\mathrm{CS}+\text { t.g. }}=S_{\mathrm{YM}} \chi+\Gamma_{\mathrm{CS}}[A+k \sigma]+\frac{1}{2} \int_{M_{3}} \operatorname{Tr} i_{\gamma}(\tilde{A}) \tilde{A}
$$

\section{The supersymmetric point}

The quantum partition function of the topological YM + CS system in presence of topological gravity backgrounds ${ }^{5}$

$$
Z\left[g_{\mu \nu}, \psi_{\mu \nu}, \gamma^{\mu}\right]=\int[d A d \tilde{A} d \tilde{c} d \sigma d \zeta] \mathrm{e}^{-\Gamma_{\mathrm{YM}+\mathrm{CS}+\mathrm{t.g}}},
$$

is an equivariant functional of the topological gravity multiplet. This means that it is both independent of $\xi^{\mu}$ and invariant under reparametrizations. At classical level it satisfies the Ward identity

$$
s Z\left[g_{\mu \nu}, \psi_{\mu \nu}, \gamma^{\mu}\right]=S Z\left[g_{\mu \nu}, \psi_{\mu \nu}, \gamma^{\mu}\right]=0,
$$

which can be - and actually is - broken by quantum anomalies. We postpone the discussion regarding quantum topological anomalies to section 6 .

We can now look for bosonic backgrounds which are left invariant by $S$ :

$$
\bar{\psi}_{\mu \nu}=0, \quad \mathcal{L}_{\bar{\gamma}} \bar{g}^{\mu \nu} \equiv \bar{D}^{\mu} \bar{\gamma}^{\nu}+\bar{D}^{\nu} \bar{\gamma}^{\mu}=0
$$

The second equation above says that the superghost background $\bar{\gamma}^{\mu}(x)$ is a Killing vector of the 3-dimensional metric $\bar{g}_{\mu \nu}(x)$. These geometrical data define a so-called Seifert structure on a 3-dimensional manifold [8]. We see therefore that supersymmetric YM+CS theories admitting a rigid topological supersymmetry are precisely those defined on 3-dimensional Seifert manifolds. Hence one anticipates that supersymmetric YM+CS theories on Seifert manifolds enjoy localization properties [4]. This fact, originally discovered in a "phenomenological" way in $[6,7]$, has been subsequently explained using various approaches by different authors $[8,10]$. In our approach this follows straighforwardly from the BRST trasformations of topological gravity.

\footnotetext{
${ }^{5}$ The notation in (4.1) is schematic: we did not include explicitly the ghost sector which fixes the standard YM gauge invariance.
} 
The topological YM+CS action on a fixed Seifert manifold

$$
\begin{gathered}
\bar{\Gamma}=S_{\mathrm{YM}} \chi+\Gamma_{\mathrm{CS}}[A+k \sigma]+\int_{M_{3}} \operatorname{Tr}\left[\frac{1}{2} i_{\bar{\gamma}}(\tilde{A}) \tilde{A}+\Lambda * i_{\bar{\gamma}}(* \tilde{A})+b * i_{\bar{\gamma}}\left(* i_{\bar{\gamma}}(\tilde{c}+k d k \sigma)\right)\right. \\
\left.+b * i_{\bar{\gamma}}(*(F+D(k \sigma)))\right]
\end{gathered}
$$

is therefore invariant under the following BRST transformations which encode both gaugeinvariance and global topological supersymmetry

$$
\begin{aligned}
S_{\mathrm{YM}} c & =-c^{2}+i_{\bar{\gamma}}(A)+\sigma, \\
S_{\mathrm{YM}} A & =-D c+i_{\bar{\gamma}}(\tilde{A})+k \zeta, \\
S_{\mathrm{YM}} \tilde{A} & =-[c, \tilde{A}]-F+i_{\bar{\gamma}}(\tilde{c})+k D \sigma, \\
S_{\mathrm{YM}} \tilde{c} & =-[c, \tilde{c}]-D \tilde{A}-[\sigma, \tilde{A}]+k d k \zeta, \\
S_{\mathrm{YM}} \sigma & =-[c, \sigma]+\zeta, \\
S_{\mathrm{YM}} \zeta & =-[c, \zeta]+i_{\bar{\gamma}}(D \sigma) .
\end{aligned}
$$

\section{The relation between topological and physical supersymmetry}

The rigid topological theory that we obtained by coupling topological YM+CS to topological gravity computes certain (semi)-topological observables of the "physical" globally supersymmetric YM+CS theory living on the same manifold. In particular the topological partition function, which is the object that we consider in this paper, is the same as the superpartition function of the "physical" theory, i.e. the partition function with supersymmetric boundary conditions on both bosons and fermions. Indeed, as we mentioned in the introduction, the almost totality of the computations of those (semi)-topological observables performed in recent years, were developed directly in the context of the "physical" theory with spinorial supercharges. We argued in the introduction that the topological gravity viewpoint provides some benefits, both conceptual and practical. For starters, we just saw in the previous section that the Seifert condition emerges from topological gravity directly - without the necessity to go through covariantly constant spinors [10], add extra symmetries [8], or pick up ingenious gauges [9]. But, above all, the coupling to topological gravity will allow us, in the next sections, to compute the moduli dependence of the partition function of supersymmetric YM+CS theory (involving only vector multiplets) by solving the anomalous topological Ward identities, in a completely regularization and gauge independent way.

In this section we will describe more precisely the relation between the topological YM+CS obtained via the coupling to topological gravity and "physical", spinorial, supersymmetric theory. We will also elucidate how the action that emerges from topological gravity encompasses the topological actions which were introduced in either [8] or [31].

Supersymmetric CS (SCS) theory on curved space has been studied starting from [10], who considered the special example of $S^{3}$. The supersymmetric extension of the CS action 
in flat space [29] writes

$$
\Gamma_{\mathrm{SCS}}=\Gamma_{\mathrm{CS}}+\int d^{3} x \operatorname{Tr}\left(D \sigma-\frac{1}{2} \lambda^{\dagger} \lambda\right)
$$

where the scalars $D, \sigma$ and the Dirac spinor $\lambda$ are in the adjoint of the gauge group. Since $D, \sigma$ and $\lambda$ are auxiliary non-dynamical fields, supersymmetric CS theory (5.1) is physically equivalent to pure CS theory. The action (5.1) is invariant under the global supersymmetry transformations which have the structure

$$
\delta \equiv \delta_{\epsilon}+\delta_{\bar{\epsilon}}
$$

where

$$
\begin{aligned}
\delta_{\epsilon} A_{\mu} & =-\frac{i}{2} \lambda^{\dagger} \gamma_{\mu} \epsilon, \\
\delta_{\epsilon} \sigma & =-\frac{1}{2} \lambda^{\dagger} \epsilon, \\
\delta_{\epsilon} D & =-\frac{i}{2} D_{\mu} \lambda^{\dagger} \gamma^{\mu} \epsilon+\frac{i}{2}\left[\lambda^{\dagger}, \sigma\right] \epsilon, \\
\delta_{\epsilon} \lambda & =-i \sqrt{g} \epsilon_{\mu \nu \rho} \gamma^{\rho} F^{\mu \nu}-D \epsilon+i \gamma^{\mu} D_{\mu} \sigma \epsilon, \\
\delta_{\epsilon} \lambda^{\dagger} & =0,
\end{aligned}
$$

and $\delta_{\bar{\epsilon}} \Phi=\left(\delta_{\epsilon} \Phi\right)^{\dagger}$, for any field $\Phi \in\left\{A, \sigma, D, \lambda, \lambda^{\dagger}\right\}$.

The classical action of ordinary, non supersymmetric, CS action has the peculiarity of being invariant under local diffeomorphisms without the need of introducing a space-time metric. This means that one can study quantum CS theory on any fixed curved manifold: topological invariance of CS theory must be thought of as a global symmetry, in the sense that one does not need to integrate over space-time metrics to make sense of the quantum theory. This global symmetry is actually broken by anomalies: but, precisely because one is dealing with a global symmetry, this does not spoil the consistency of the quantum theory.

One might imagine that, by analogy, SCS theory might be made invariant under local supersymmetry transformations without the need of explicitly introducing supergravity backgrounds. If this were so, SCS could be formulated consistently on any manifolds. Let us discuss why this is not the case.

The standard recipe for putting a generic supersymmetric theory on a curved manifold is to first couple it to supergravity, by promoting the global supersymmetry transformations (5.3) to local ones. For the SCS theory, this would mean in principle to couple (5.1) to the Noether supercurrents

$$
S^{\mu}=\frac{i}{2} \lambda^{\dagger} \gamma^{\mu} \sigma, \quad \bar{S}^{\mu}=-\frac{i}{2} \gamma^{\mu} \lambda \sigma,
$$

by changing the action

$$
\Gamma_{\mathrm{SCS}}^{\text {curved }}=\Gamma_{\mathrm{SCS}}+\psi_{\mu}^{\dagger} \bar{S}^{\mu}+S^{\mu} \psi_{\mu}+\cdots
$$


where $\psi_{\mu}$ is the gravitino field and the dots denote the higher order terms of the Noether procedure. The coupled action $\Gamma_{\mathrm{SCS}}^{\mathrm{curved}}$ is invariant - at linearized level - under local supersymmetry transformations (5.3) of the fields if the gravitino background also transforms as

$$
\delta_{\epsilon} \psi_{\mu}=D_{\mu} \epsilon+\cdots, \quad \delta_{\bar{\epsilon}} \psi_{\mu}^{\dagger}=D_{\mu} \epsilon^{\dagger}+\cdots
$$

However SCS theory is "almost" topological. This is reflected by the fact that the supercurrents (5.4) vanish on shell

$$
S^{\mu}=i \alpha \frac{\delta \Gamma_{\mathrm{SCS}}}{\delta \lambda} \gamma^{\mu} \sigma+\frac{i}{2}(1-\alpha) \lambda^{\dagger} \gamma^{\mu} \frac{\delta \Gamma_{\mathrm{SCS}}}{\delta D},
$$

where $\alpha$ is an arbitrary parameter. Since, when $\epsilon$ is space-dependent, the supersymmetry variation of the flat space action writes in terms of the supercurrents as follows

$$
\begin{aligned}
\left(\delta_{\epsilon}+\delta_{\bar{\epsilon}}\right) \Gamma_{\mathrm{SCS}} & =\int d^{3} x\left(S^{\mu} D_{\mu} \epsilon+D_{\mu} \epsilon^{\dagger} \bar{S}^{\mu}\right)= \\
& =\int d^{3} x\left(i \alpha \frac{\delta \Gamma_{\mathrm{SCS}}}{\delta \lambda} \gamma^{\mu} \sigma+\frac{i}{2}(1-\alpha) \lambda^{\dagger} \gamma^{\mu} \frac{\delta \Gamma_{\mathrm{SCS}}}{\delta D}\right) D_{\mu} \epsilon+\text { c.c. },
\end{aligned}
$$

one sees there is an alternative way to make (the diffeomorphism invariant extension of) $\Gamma_{\text {SCS }}$ locally supersymmetric. This alternative method does not require introducing the gravitino: thanks to on-shell vanishing of the supercurrents one can simply modify the supersymmetry variations of $\lambda, \lambda^{\dagger}$ and $D$

$$
\begin{aligned}
\tilde{\delta}_{\epsilon} \lambda & =\delta_{\epsilon} \lambda+i \alpha \gamma^{\mu} D_{\mu} \epsilon \sigma, \\
\tilde{\delta}_{\epsilon} D & =\delta_{\epsilon} D-\frac{i}{2}(1-\alpha) \lambda^{\dagger} \gamma^{\mu} D_{\mu} \epsilon,
\end{aligned}
$$

where the covariant derivatives are those appropriate to the chosen curved manifold. Then, (5.8) is obviously equivalent to

$$
\tilde{\delta}_{\epsilon} \Gamma_{\mathrm{SCS}}=\tilde{\delta}_{\bar{\epsilon}} \Gamma_{\mathrm{SCS}}=0
$$

for space-time dependent $\epsilon$ 's.

The trouble with this "alternative" way to make the supersymmetry local is that, for $\alpha$ arbitrary and for generic manifolds, the local supersymmetry algebra does not close. By analyzing the supersymmetry commutation relations one discovers [32] that closure of the algebra requires both that the condition

$$
\alpha=\frac{2}{3}
$$

is met and that the space-time dependence of $\epsilon$ be restricted by the differential equation

$$
\gamma^{\mu} \gamma^{\nu} D_{\mu} D_{\nu} \epsilon=h \epsilon .
$$

One concludes that CS theory with rigid supersymmetry can be constructed only on manifolds for which solutions of eq. (5.12) exist. For those special manifolds one can obtain 
the corresponding rigid supersymmetry transformations by replacing in (5.9) the spinors which solve eq. (5.12).

The lesson of this discussion is that, even for the "almost topological" supersymmetric CS theories one cannot neglect the coupling of the (classically vanishing) supercurrents to the supergravity backgrounds. Indeed it has since been understood [5, 30] that the conditions (5.11) and (5.12) are to be interpreted, in a model independent way, as the equations for the vanishing of the supersymmetry variation of the gravitino background

$$
\delta_{\epsilon} \psi_{\mu}=\delta_{\bar{\epsilon}} \psi_{\mu}^{\dagger}=0 \text {. }
$$

The nice feature of eqs. (5.13), which are readily seen to imply (5.12), is their universality: they do not depend on the specific theory one is considering and they characterize manifolds on which field theories with global supersymmetry may be constructed. The specific form of supersymmetry does instead depend on both the solution of (5.13) and the form of the coupling of the supergravity multiplet to the theory at hand.

It can be shown that in the real case when a solution $\epsilon$ of $\delta_{\epsilon} \psi_{\mu}=0$ defines by conjugation a solution of $\delta_{\bar{\epsilon}} \psi_{\mu}^{\dagger}=0$, the vector

$$
\bar{\gamma}^{\mu}=\epsilon^{\dagger} \Gamma^{\mu} \epsilon
$$

is a (real) Killing vector of the underlying 3-manifold

$$
D_{\mu} \bar{\gamma}_{\nu}+D_{\nu} \bar{\gamma}_{\mu}=0
$$

This explains in particular why CS theories on 3-manifolds admitting a U(1) action known as Seifert 3-manifolds - enjoy the localization property which was originally discovered in $[6,7]$ in an experimental way. We have seen that in our topological approach, the Seifert condition (5.15) emerged directly from the topological gravity BRST transformation laws, with no reference to (generalized) covariantly constant spinors.

We can consider also the supersymmetric YM action in the SCS theory:

$$
\Gamma_{\mathrm{SCS}+\mathrm{SYM}}=\Gamma_{\mathrm{SCS}}+\Gamma_{\mathrm{SYM}}
$$

where $^{6}$

$$
\Gamma_{\mathrm{SYM}}=\operatorname{Tr}\left[\frac{1}{4} F_{\mu \nu}^{2}+\frac{1}{2} D_{\mu} \sigma D^{\mu} \sigma+\frac{1}{2}\left(D+\frac{\sigma}{r}\right)^{2}+\frac{i}{2} \lambda^{\dagger} \gamma^{\mu} D_{\mu} \lambda+\frac{i}{2} \lambda^{\dagger}[\sigma, \lambda]-\frac{1}{4 r} \lambda^{\dagger} \lambda\right] .
$$

This latter action is not only invariant under the global supersymmetry transformations (5.3), (5.9), (5.13), but also supersymmetric exact

$$
\epsilon^{\dagger} \epsilon \mathcal{L}_{\mathrm{YM}}=\tilde{\delta}_{\epsilon} \tilde{\delta}_{\bar{\epsilon}} \operatorname{Tr} \frac{1}{4}\left(\lambda^{\dagger} \lambda-2 D \sigma\right) .
$$

Therefore, cohomologically, the SCS+SYM system is equivalent to SCS theory.

\footnotetext{
${ }^{6}$ The parameter $r$ which appears in this formula is the radius of the $S^{1}$ of the Seifert fibration.
} 
The supersymmetric SCS+SYM on a fixed Seifert manifold can be twisted to give a model with a topological rigid symmetry. This was done in [31]. The physical supersymmetric vector multiplet (5.3) includes a Dirac fermion $\lambda$ which has 4 real components. After the twist of [31], three of those form the topological gaugino $\Psi$. This, together with the scalar $\sigma$ and the gauge connection $A$ form the multiplet of topological YM. The twisted supersymmetry transformations of this supermultiplet turn out to have the form (3.8), in which the topological gravity field $\gamma^{\mu}$ is replaced by the Reeb vector field $\bar{\gamma}^{\mu}$. The remaining fermion gives rise to a scalar $\alpha$ of ghost number +1 which form, together with the auxiliary scalar field D of ghost number +2 , an additional BRST trivial doublet,

$$
\begin{aligned}
S_{\mathrm{YM}} \alpha & =-[c, \alpha]+\mathrm{D}+X(A, \sigma), \\
S_{\mathrm{YM}} \mathrm{D} & =-[c, \mathrm{D}]+i_{\bar{\gamma}} D \alpha+[\sigma, \alpha]-\tilde{S} X(A, \sigma) .
\end{aligned}
$$

Note that $S_{\mathrm{YM}}$ is nilpotent for any choice of the scalar function $X(A, \sigma)$ of ghost number 2. The twist of the physical supersymmetric theory gives rise to a specific choice for $X(A, \sigma)$. We keep it arbitrary for the moment, to better explain the connections with other approaches.

The additional BRST trivial doublet $(\alpha, \mathrm{D})$ can function as an antighost-Lagrange multiplier pair, by adding to the $S_{\mathrm{YM}}$ invariant action (3.26) an $S_{\mathrm{YM}}$-trivial term

$$
\Gamma_{\mathrm{CS}}^{\prime}=\Gamma_{\mathrm{CS}}[A+k \sigma]+\frac{1}{2} \int_{M_{3}} k \operatorname{Tr} \Psi \Psi+S_{\mathrm{YM}} \int_{M_{3}} k d k \operatorname{Tr} \alpha \sigma
$$

One can pick

$$
X(A, \sigma)=0
$$

With this choice the gauge-fixing term in (5.20) fixes the Beasley-Witten shift-symmetry: integrating out D puts $\sigma$ to zero and integrating out $\alpha$ sets $\zeta=0$ and thus $\Psi=i_{\gamma}(\tilde{A})$. We recover in this way our original CS action (2.14).

The twist of the physical SCS action (5.1) discussed in [31] gives instead the

$$
X(A, \sigma)=\frac{k F}{k d k}+\sigma=i_{\gamma}(* F)+\sigma .
$$

Note that the choice of $X(A, \sigma)$ introduces a spurious dependence of the BRST operator on the metric compatible with the vector field $\bar{\gamma}^{\mu}$ which defines the Seifert structure. This dependence should of course drop out in physical observables, but this is not explicit in the framework of [31]. The reason of course is that twisting a physical supersymmetric action corresponds to make a specific choice for the gauge-fixing term of the topological action. This, although sometimes convenient to perform explicit computations, leads to gauge-dependent BRST transformations, somehow obscuring the geometric content of the topological symmetry. One appealing feature of our treatment is that it makes manifest that the theory only depends on the Seifert structure. 


\section{Topological Anomaly for Seifert manifolds}

The classical Ward identity (4.2) can be broken by quantum anomalies

$$
S \log Z\left[g_{\mu \nu}, \psi_{\mu \nu}, \gamma^{\mu}\right] \equiv S \int_{M_{3}} i \Gamma\left[g_{\mu \nu}\right]=\frac{c}{6} \int_{M_{3}} i A_{1}^{(3)}\left[g_{\mu \nu}, \psi_{\mu \nu}\right] .
$$

where $c$ is the anomaly coefficient. The topological anomaly describes therefore the response of the quantum action density $\Gamma\left[g_{\mu \nu}\right]$ of the $\mathrm{YM}+\mathrm{CS}$ topological system under a generic variation of the metric $\delta g_{\mu \nu} \equiv \psi_{\mu \nu}$

$$
\delta \int_{M_{3}} \Gamma\left[g_{\mu \nu}\right]=\frac{c}{6} \int_{M_{3}} A_{1}^{(3)}\left[g_{\mu \nu}, \delta g_{\mu \nu}\right]
$$

The topological anomaly 3 -form $A_{1}^{(3)}$ is a local cohomology class of ghost number +1 of the BRST operator of topological gravity, which must satisfy the Wess-Zumino consistency condition

$$
S A_{1}^{(3)}\left[g_{\mu \nu}, \psi_{\mu \nu}\right]=-d A_{2}^{(2)}\left[g_{\mu \nu}, \psi_{\mu \nu}, \gamma^{\mu}\right] .
$$

Topological anomalies in any dimensions were classified in [22]. In 3 dimensions we have a single representative of ghost number +1

$$
A_{1}^{(3)}\left[g_{\mu \nu}, \psi_{\mu \nu}\right]=\epsilon^{\mu \nu \rho} R_{\mu}^{\alpha} D_{\nu} \psi_{\rho \alpha} d^{3} x
$$

From the structure of the anomaly, it is clear that the parity invariant topological YM part of the action cannot contribute to $c$. A non-trivial $c$ can only come from the CS part $\Gamma_{\mathrm{CS}+\text { t.g. }}$ of the action. Since this theory is equivalent to bosonic CS, we conclude that $c$ is nothing but the coefficient of the framing anomaly of pure bosonic CS. For $\mathrm{SU}(N)$ gauge theories this has been computed in $[13]:^{7}$

$$
c_{\mathrm{SU}(N)}=\frac{1}{4 \pi} \frac{N^{2}-1}{k}
$$

3-dimensional diffeomorphisms are not anomalous. Hence, there exists a renormalization prescription which gives rise to an effective (non-local) action $\Gamma\left[g_{\mu \nu}\right]$ which transforms as a 3-form under 3-dimensional generic diffeomorphisms. To express this condition, it is useful to introduce the Bardeen-Zumino BRST operator $S_{\text {diff }}$ [33] associated to 3dimensional diffeomorphisms:

$$
S_{\text {diff }}=\mathcal{L}_{\xi}-\left\{i_{\xi}, d\right\}, \quad S_{\text {diff }}^{2}=0,
$$

where $\xi=\xi^{\mu} \partial_{\mu}$ is the reparametrization ghost in 3-dimensions, and $\mathcal{L}_{\xi}$ denotes the action of the Lie derivative along $\xi$ on the metric $g_{\mu \nu}$. The equation

$$
S_{\text {diff }} \Gamma\left[g_{\mu \nu}\right]=0,
$$

\footnotetext{
${ }^{7}$ In this section we introduced a factor $\frac{k}{2 \pi}$ in the normalization of the Chern-Simons action, to make it gauge-invariant modulo $2 \pi$. Notice also that the parameter $k$ which appears in this and in the following formulas should be taken as the "shifted" $l+2$, with respect to the level $l$ of the Kac-Moody current algebra whose conformal blocks map to the states obtained from canonically quantizing the theory.
} 
precisely expresses the fact that the quantum action density $\Gamma\left[g_{\mu \nu}\right]$ transforms as 3 -form under reparametrizations.

After these preliminaries, let us now make our main observation: when considering YM+CS topological theories on Seifert manifolds, one relaxes the request (6.7) of full 3-dimensional reparametrization invariance. One is satisfied with invariance under reparametrizations which preserve the Seifert structure: these are reparametrizations whose ghost fields $\xi^{\mu}$ commute with the Reeb vector $\bar{\gamma}^{\mu}$. Let us denote by

$$
S_{\text {diff }}^{\text {Seif }}=\mathcal{L}_{\xi_{\text {Seif }}}-\left\{i_{\xi_{\text {Seif }}}, d\right\}, \quad \mathcal{L}_{\xi_{\text {Seif }}} \bar{\gamma}=0,
$$

the Bardeen-Zumino BRST operator associated to diffeomorphisms preserving $\bar{\gamma}^{\mu}$. One also restricts the topological gravity background fields to those left invariant under $\mathcal{L}_{\bar{\gamma}}$

$$
\mathcal{L}_{\bar{\gamma}} g_{\mu \nu}=\mathcal{L}_{\bar{\gamma}} \psi_{\mu \nu}=\mathcal{L}_{\bar{\gamma}} \gamma^{\mu}=0
$$

To parametrize solutions of (6.9) it is useful to introduce systems of coordinates adapted to the Seifert structure associated to $\bar{\gamma}^{\mu}$ :

$$
\begin{aligned}
& (d s)_{M}^{2}=\mathrm{e}^{\sigma} k \otimes k+g_{i j} d x^{i} \otimes d x^{j}= \\
& =\mathrm{e}^{\sigma} d y \otimes d y+2 \mathrm{e}^{\sigma} a_{i} d x^{i} \otimes d y+\left(g_{i j}+\mathrm{e}^{\sigma} a_{i} a_{j}\right) d x^{i} \otimes d x^{j},
\end{aligned}
$$

where $k$ is the contact 1 -form

$$
k \equiv d y+a_{i} d x^{i}
$$

dual to the Reeb vector field

$$
i_{\bar{\gamma}}(k)=1,
$$

$\sigma, g_{i j}$ and $a_{i}$ are fields on the two-dimensional surface $\Sigma_{2}$, associated to the Seifert fibration $\pi: M \rightarrow \Sigma_{2}$. The invariant $\psi_{\mu \nu}$ are analogously parametrized by fermions $\zeta, \psi_{i j}$ and $\psi_{i}$ living on $\Sigma_{2}$, defined as follows:

$$
\psi_{\mu \nu}=\left(\begin{array}{cc}
\mathrm{e}^{\sigma} \zeta & \mathrm{e}^{\sigma} \psi_{i}+\mathrm{e}^{\sigma} \zeta a_{i} \\
\mathrm{e}^{\sigma} \psi_{i}+\mathrm{e}^{\sigma} \zeta a_{i} & \psi_{i j}+\mathrm{e}^{\sigma}\left(\psi_{i} a_{j}+\psi_{j} a_{i}+\zeta a_{i} a_{j}\right)
\end{array}\right) .
$$

Finally, the invariant $\xi^{\mu}$ and $\gamma^{\mu}$ can be written in terms of fields living on $\Sigma_{2}$ as

$$
\xi^{\mu}=\left(\xi^{0}, \xi^{i}\right) \equiv\left(\xi^{0}, \vec{\xi}\right), \quad \gamma^{\mu}=\left(\gamma^{0}, \gamma^{i}\right) \equiv\left(\gamma^{0}, \vec{\gamma}\right) .
$$

Therefore, in the Seifert case, the effective action $\Gamma^{\mathrm{Seif}}\left[g_{i j}, \sigma, a_{i}\right]$ is a functional of the fields $\sigma, g_{i j}$ and $a_{i}$, and the appropriate renormalization prescription writes

$$
S_{\text {diff }}^{\text {Seif }} \Gamma^{\text {Seif }}\left[g_{i j}, \sigma, a_{i}\right]=0
$$

The action density $\Gamma\left[g_{\mu \nu}\right]$ which satisfies the (strong) prescription (6.7) defines, of course, once written in Seifert adapted coordinates, also an action density $\Gamma^{\operatorname{Seif}}\left[g_{i j}, \sigma, a_{i}\right]$ satisfiying the (weaker) Seifert renormalization condition (6.15):

$$
\Gamma^{\text {Seif }}\left[g_{i j}, \sigma, a_{i}\right]=\left.\Gamma\left[g_{\mu \nu}\right]\right|_{\mathcal{L}_{\bar{\gamma}} g_{\mu \nu}=0} .
$$


This effective action satisfies the topological anomaly equation

$$
s_{\text {Seif }} \int_{M_{3}} \Gamma^{\text {Seif }}\left[g_{i j}, \sigma, a_{i}\right]=\frac{c}{6} \int_{M_{3}} A_{1}^{(3)}\left[g_{i j}, \sigma, a_{i} ; \psi_{i j}, \zeta, \psi_{i}\right],
$$

where the r.h.s. is obtained from $A_{1}^{(3)}\left[g_{\mu \nu}, \psi_{\mu \nu}\right]$ by evaluating it for $\bar{\gamma}$-invariant fields (6.9). The BRST operator $s_{\text {Seif }}$ in the l.h.s. of the equation above encodes topological gravity transformations which preserves the Seifert structure

$$
\begin{aligned}
s_{\text {Seif }} \xi^{i} & =-\frac{1}{2} \mathcal{L}_{\vec{\xi}} \xi^{i}+\gamma^{i}, & s_{\text {Seif }} \gamma^{i} & =-\mathcal{L}_{\vec{\xi}} \gamma^{i}, \\
s_{\text {Seif }} g_{i j} & =-\mathcal{L}_{\vec{\xi}} g_{i j}+\psi_{i j}, & s_{\text {Seif }} \psi_{i j} & =-\mathcal{L}_{\vec{\xi}} \psi_{i j}+\mathcal{L}_{\vec{\gamma}} g_{i j}, \\
s_{\text {Seif }} \xi^{0} & =-\mathcal{L}_{\vec{\xi}} \xi^{0}+\gamma^{0}, & s_{\text {Seif }} \gamma^{0} & =-\mathcal{L}_{\vec{\xi}} \gamma^{0}+\mathcal{L}_{\vec{\gamma}} \xi^{0}, \\
s_{\text {Seif }} a_{i} & =-\mathcal{L}_{\vec{\xi}} a_{i}-\partial_{i} \xi^{0}+\psi_{i}, & s_{\text {Seif }} \psi_{i} & =-\mathcal{L}_{\vec{\xi}} \psi_{i}+\partial_{i} \gamma^{0}+\mathcal{L}_{\vec{\gamma}} a_{i}, \\
s_{\text {Seif }} \sigma & =-\mathcal{L}_{\vec{\xi}} \sigma+\zeta, & s_{\text {Seif }} \zeta & =-\mathcal{L}_{\vec{\xi}} \zeta+\mathcal{L}_{\vec{\gamma}} \sigma .
\end{aligned}
$$

The invariant gravitational background fields split into three multiplets: one is the 2dimensional topological gravity multiplet $\left(\xi^{i}, \gamma^{i}, g_{i j}, \psi_{i j}\right)$. Then there is an abelian topological gauge multiplet $\left(\xi^{0}, \gamma^{0}, a_{i} \psi_{i}\right)$ : their BRST properties are not just the "flat" ones, but they are modified by the coupling to 2-dimensional gravity. Finally there is also an uncharged scalar topological multiplet $(\sigma, \zeta)$ : this too is coupled to 2-dimensional topological gravity.

Writing $A_{1}^{(3)}\left[g_{i j}, \sigma, a_{i} ; \psi_{i j}, \zeta, \psi_{i}\right]$ in adapted coordinates

$$
A_{1}^{(3)}\left[g_{i j}, \sigma, a_{i} ; \psi_{i j}, \zeta, \psi_{i}\right]=\frac{1}{2} \mathcal{A} d y \epsilon_{i j} d x^{i} d x^{j},
$$

one finds the following expression for $\mathcal{A}$ :

$$
\begin{aligned}
\mathcal{A}= & -\frac{1}{2} \sqrt{g} \psi_{i j} \mathrm{e}^{\sigma}\left[D^{i} D^{j} f+3 D^{i} f D^{j} \sigma+2 f D^{i} \sigma D^{j} \sigma+f D^{i} D^{j} \sigma\right] \\
& +\frac{1}{2} \sqrt{g} \mathrm{e}^{\sigma} \psi_{i}^{i}\left[D^{2} f+f\left(\frac{1}{2} R_{2}-\mathrm{e}^{\sigma} f^{2}\right)\right. \\
& \left.\quad+\frac{5}{2} D_{j} f D^{j} \sigma+\frac{3}{2} f D_{j} \sigma D^{j} \sigma+\frac{3}{2} f D^{2} \sigma\right] \\
& +\frac{1}{2} \epsilon^{i j} \mathrm{e}^{\sigma} \psi_{i}\left[6 \mathrm{e}^{\sigma} f D_{j} f-D_{j} R_{2}\right. \\
& \left.+6 \mathrm{e}^{\sigma} f^{2} D_{j} \sigma-R_{2} D_{j} \sigma-D_{j} D^{2} \sigma-D_{j} \sigma D^{2} \sigma\right] \\
& +\sqrt{g} \mathrm{e}^{\sigma} \zeta\left[\mathrm{e}^{\sigma} f^{3}-\frac{1}{2} f R_{2}-D_{i} f D^{i} \sigma-\frac{1}{2} f D_{i} \sigma D^{i} \sigma-f D^{2} \sigma-\frac{1}{2} D^{2} f\right] .
\end{aligned}
$$

In (6.20), $R_{2}$ is the scalar curvature constructed with the 2-dimensional metric $g_{i j}$ and

$$
f=\frac{\epsilon^{i j}}{\sqrt{g}} f_{i j}=\frac{\epsilon^{i j}}{\sqrt{g}}\left(\partial_{i} a_{j}-\partial_{j} a_{i}\right)
$$

is the scalar field dual to the $\mathrm{U}(1)$ field strength $f^{(2)} \equiv d a$. 
The important fact, now, is that $A_{1}^{(3)}\left[g_{i j}, \sigma, a_{i} ; \psi_{i j}, \zeta, \psi_{i}\right]$ is $s_{\text {Seif }}$-trivial

$$
\mathcal{A}=s_{\text {Seif }} \Gamma_{\mathrm{WZ}}^{\mathrm{Seif}}\left[g_{i j}, \sigma, a_{i}\right],
$$

where the Wess-Zumino action $\Gamma_{\mathrm{WZ}}^{\text {Seif }}$ is the following local functional ${ }^{8}$

$$
\Gamma_{\mathrm{WZ}}^{\mathrm{Seif}}\left[g_{i j}, \sigma, a_{i}\right]=\frac{1}{2} \sqrt{g} \mathrm{e}^{2 \sigma} f^{3}-\sqrt{g} \frac{1}{2} \mathrm{e}^{\sigma} f R_{2}-\frac{1}{2} \sqrt{g} \mathrm{e}^{\sigma} f D^{2} \sigma .
$$

$\Gamma_{\mathrm{WZ}}^{\mathrm{Seif}}\left[g_{i j}, \sigma, a_{i}\right]$ is a legitimate Wess-Zumino action since it is both local and invariant under reparametrizations which preserve the Seifert structure

$$
S_{\text {diff }}^{\text {Seif }} \Gamma_{\mathrm{WZ}}^{\text {Seif }}\left[g_{i j}, \sigma, a_{i}\right]=0 .
$$

It should be kept in mind, however, that $\Gamma_{\mathrm{WZ}}^{\mathrm{Seif}}\left[g_{i j}, \sigma, a_{i}\right]$ - unlike the non-local $\Gamma\left[g_{\mu \nu}\right]$ in eq. (6.16) - is not invariant under the full 3 -dimensional $S_{\text {diff }}$.

Hence one can define the effective action

$$
\tilde{\Gamma}^{\text {Seif }}\left[g_{i j}, \sigma, a_{i}\right] \equiv \Gamma^{\operatorname{Seif}}\left[g_{i j}, \sigma, a_{i}\right]-\frac{c}{6} \Gamma_{\mathrm{WZ}}^{\operatorname{Seif}}\left[g_{i j}, \sigma, a_{i}\right],
$$

which is both $s_{\text {Seif }}$-invariant - i.e. topological in the Seifert sense -

$$
s_{\text {Seif }} \tilde{\Gamma}^{\text {Seif }}\left[g_{i j}, \sigma, a_{i}\right]=0,
$$

and invariant under reparametrizations which preserve $\bar{\gamma}$

$$
S_{\text {diff }}^{\text {Seif }} \tilde{\Gamma}^{\text {Seif }}\left[g_{i j}, \sigma, a_{i}\right]=0,
$$

Summarizing, we have shown that it is always possible to define through eq. (6.25) a quantum action density $\tilde{\Gamma}^{\text {Seif }}\left[g_{i j}, \sigma, a_{i}\right]$ which depends on the moduli parametrizing the Seifert structures (which we will characterize in section 7 ) but not on the specific adapted metric which one picks to quantize the theory.

\section{$7 \quad$ Moduli}

In this section we will identify cohomologically the parameters on which the quantum partition function of $3 \mathrm{~d}$ supersymmetric gauge theory depends.

In the general "non-real" case [5], the space of infinitesimal deformations of a given supersymmetric background can be identified $[34,35]$ with the appropriate cohomology of a certain differential operator $\tilde{\partial}$ which, loosely speaking, is the $3 \mathrm{~d}$ generalization of the

\footnotetext{
${ }^{8}$ The topological anomaly $A_{1}^{(3)}$ satisfies also: $A_{1}^{(3)}=S \Gamma_{\mathrm{GCS}}^{(3)}[g]$, where $\Gamma_{\mathrm{GCS}}[g]$ is the gravitational ChernSimons action. In [8] it is pointed out that on a Seifert manifold $M$ there exists a natural trivialization of the double tangent $T M \oplus T M$ which leads to a specific definition of the Chern-Simons invariant $\Gamma_{\mathrm{GCS}}[g]$. This provides a geometric explanation of why the topological anomaly is BRST trivial in the Seifert context. To the best of our knowledge however an explicit local Wess-Zumino functional of the adapted Seifert metric as $\Gamma_{\mathrm{WZ}}^{\mathrm{Seif}}\left[g_{i j}, \sigma, a_{i}\right]$ has not been presented earlier. Note that $\Gamma_{\mathrm{GCS}}^{(3)}[g]$ evaluated for an adapted Seifert metric and $\Gamma_{\mathrm{WZ}}^{\text {Seif }}\left[g_{i j}, \sigma, a_{i}\right]$ differ by a not-globally defined total derivative, whose precise form is described in appendix $\mathrm{B}$.
} 
2-dimensional Dolbeault operator $\bar{\partial}$. The cohomology of $\tilde{\partial}$ has not been well studied yet, and, to our knowledge, it is not explicitly known for general 3-manifolds. On the other hand, in the more special "real" context to which our analysis is confined one can give a very explicit and general characterization of the space of infinitesimal deformations of (topological) supersymmetric backgrounds.

We should also emphasize that the analysis of topological quantum anomalies contained in the previous section makes our identification of the parameters which affect the partition function valid both at the non-linear and the quantum level.

We have seen that supersymmetric topological backgrounds correspond to solutions of the Killing equations

$$
\mathcal{L}_{\bar{\gamma}} \bar{g}_{\mu \nu} \equiv \bar{D}_{\mu} \bar{\gamma}_{\nu}+\bar{D}_{\nu} \bar{\gamma}_{\mu}=0
$$

Given a solution $\left\{\bar{g}_{\mu \nu}, \bar{\gamma}^{\mu}\right\}$ of (7.1) we want explore nearby supersymmetric backgrounds $\left\{\bar{g}_{\mu \nu}+\delta g_{\mu \nu}, \gamma^{\mu}+\delta \gamma^{\mu}\right\}$. The deformations $\left\{\delta g_{\mu \nu}, \delta \gamma^{\mu}\right\}$ must satisfy the linear equation

$$
\mathcal{L}_{\bar{\gamma}} \delta g_{\mu \nu}+\mathcal{L}_{\delta \gamma} \bar{g}_{\mu \nu}=0
$$

Let us introduce the vector space

$$
V_{0}=\Gamma\left(T M_{3}\right) \oplus \operatorname{Sym}_{2}\left(T M_{3}\right),
$$

where $\Gamma\left(T M_{3}\right)$ is the space of vector fields on $M_{3}$ and $\operatorname{Sym}_{2}\left(T M_{3}\right)$ the space of 2-index symmetric tensors on $M_{3}$. The deformation equation (7.2) describes therefore the kernel of the linear operator

$$
\begin{aligned}
& Q_{0}: V_{0} \rightarrow V_{1} \\
& Q_{0}:\left(\delta \gamma^{\mu}, \delta g_{\mu \nu}\right) \rightarrow \mathcal{L}_{\bar{\gamma}} \delta g_{\mu \nu}+\mathcal{L}_{\delta \gamma} \bar{g}_{\mu \nu},
\end{aligned}
$$

where

$$
V_{1}=\operatorname{Sym}_{2}\left(T M_{3}\right)
$$

We are interested in characterizing physical deformations, i.e. solutions of this equation modulo gauge equivalences. Gauge-invariance includes infinitesimal diffeomorphisms

$$
\left(\delta \gamma^{\mu}, \delta g_{\mu \nu}\right) \sim\left(\delta \gamma^{\mu}, \delta g_{\mu \nu}\right)+\left(\mathcal{L}_{\xi} \delta \gamma^{\mu}, \mathcal{L}_{\xi} \delta g_{\mu \nu}\right)
$$

where $\xi^{\mu}$ is a vector field on $M_{3}$. But $\mathcal{L}_{\bar{\gamma}}$-invariant topological deformations of the metric should also be treated as a gauge invariances

$$
\left(\delta \gamma^{\mu}, \delta g_{\mu \nu}\right) \sim\left(\delta \gamma^{\mu}, \delta g_{\mu \nu}+\psi_{\mu \nu}\right)
$$

for any $\mathcal{L}_{\bar{\gamma}}$-invariant $\psi_{\mu \nu}{ }^{9}$

$$
\psi_{\mu \nu} \in \operatorname{Sym}_{2}^{i n v}\left(T M_{3}\right) \equiv\left\{\psi_{\mu \nu} \in \operatorname{Sym}_{2}\left(T M_{3}\right) \mid \mathcal{L}_{\bar{\gamma}} \psi_{\mu \nu}=0\right\}
$$

\footnotetext{
${ }^{9}$ The explicit form for invariant $\psi_{\mu \nu}$, in adapted coordinates, is given in eq. (6.13).
} 
We can therefore define a linear operator $Q_{-1}$ which captures both gauge equivalences (7.6) and (7.7)

$$
\begin{aligned}
& Q_{-1}: V_{-1} \rightarrow V_{0}, \\
& Q_{-1}:\left\{\xi^{\mu}, \psi_{\mu \nu}\right\} \rightarrow\left\{\mathcal{L}_{\xi} \bar{g}_{\mu \nu}+\psi_{\mu \nu}, \mathcal{L}_{\xi} \bar{\gamma}^{\mu}\right\},
\end{aligned}
$$

where

$$
V_{-1}=\Gamma\left(T M_{3}\right) \oplus \operatorname{Sym}_{2}^{i n v}\left(T M_{3}\right)
$$

We have

$$
Q_{0} Q_{-1}=0
$$

One can consider therefore the complex

$$
0 \rightarrow V_{-1} \stackrel{Q}{\rightarrow} V_{0} \stackrel{Q}{\rightarrow} V_{1} \rightarrow 0
$$

The associated cohomology space

$$
H_{0}(Q)=\frac{\operatorname{ker} Q_{0}}{\operatorname{Im} Q_{-1}}
$$

describes therefore inequivalent deformations around the Seifert structure $\left\{\bar{g}_{\mu \nu}, \bar{\gamma}^{\mu}\right\}$. The kernel of $Q_{-1}$

$$
\operatorname{ker} Q_{-1}=H_{-1}(Q)=\left\{\left(\xi^{\mu}, \psi_{\mu \nu}\right):[\xi, \bar{\gamma}]=0, \psi_{\mu \nu}=-\mathcal{L}_{\xi} \bar{g}_{\mu \nu}\right\}
$$

is isomorphic to the commutant $C_{\bar{\gamma}}$ of $\bar{\gamma}^{\mu}$ in the Lie algebra of vectors fields on $M_{3}$ :

$$
C_{\bar{\gamma}}=\left\{\gamma^{\mu} \in \Gamma\left(T M_{3}\right):[\gamma, \bar{\gamma}]=0\right\} \simeq H_{-1}(Q) .
$$

Consider now the map between $C_{\bar{\gamma}}$ and $\operatorname{Sym}_{2}^{\text {inv }}\left(T M_{3}\right)$ :

$$
\begin{aligned}
& \varphi: C_{\bar{\gamma}} \rightarrow \operatorname{Sym}_{2}^{i n v}\left(T M_{3}\right), \\
& \varphi: \gamma^{\mu} \rightarrow \mathcal{L}_{\gamma} \bar{g}_{\mu \nu} .
\end{aligned}
$$

The kernel of $\varphi$ is made of the isometries of $\bar{g}_{\mu \nu}$ which commute with $\bar{\gamma}^{\mu}$

$$
\operatorname{ker} \varphi=\left\{\gamma:[\gamma, \bar{\gamma}]=0, \mathcal{L}_{\gamma} \bar{g}_{\mu \nu}=0\right\} \subset C_{\bar{\gamma}} .
$$

The cokernel of $\varphi$ is, on the other hand, characterized by the $\mathcal{L}_{\bar{\gamma}}$-invariant $\psi_{\mu \nu}$ 's which are orthogonal to $\operatorname{Img} \varphi$

$$
0=\int_{M_{3}} \gamma^{\nu} \bar{D}^{\mu} \psi_{\mu \nu}=\int_{M_{3}} \gamma^{\nu} g_{\nu \mu} v^{\mu} \equiv\langle\gamma, v\rangle \quad \forall \gamma \in C_{\bar{\gamma}}
$$

where

$$
v^{\mu} \equiv \bar{g}^{\mu \lambda} \bar{D}^{\nu} \psi_{\lambda \nu}
$$


The vector $v^{\mu}$ is $\mathcal{L}_{\bar{\gamma}}$-invariant, since $\bar{g}_{\mu \nu}$ and $\psi_{\mu \nu}$ are:

$$
\mathcal{L}_{\bar{\gamma}} v^{\mu}=0=[\bar{\gamma}, v] .
$$

Hence $v^{\mu} \in C_{\bar{\gamma}}$. But since, according to (7.18) $v^{\mu}$ is orthogonal to whole $C_{\bar{\gamma}}$, it vanishes

$$
v^{\mu}=\bar{g}^{\mu \lambda} \bar{D}^{\nu} \psi_{\lambda \nu}=0 .
$$

We conclude that

$$
\operatorname{coker} \varphi=\left\{\psi_{\mu \nu}: \mathcal{L}_{\bar{\gamma}} \psi_{\mu \nu}=0, \bar{D}^{\mu} \psi_{\mu \nu}=0\right\}
$$

and therefore

$$
\operatorname{Sym}_{2}^{i n v}\left(T M_{3}\right)=\operatorname{Im} \varphi \oplus \operatorname{coker} \varphi \simeq \frac{C_{\bar{\gamma}}}{\operatorname{ker} \varphi} \oplus \operatorname{coker} \varphi
$$

Let us now consider the cokernel of $Q_{0}$ : it is characterized by the equation

$$
\int_{M_{3}} \psi^{\mu \nu}\left(\mathcal{L}_{\bar{\gamma}} \delta g_{\mu \nu}+\mathcal{L}_{\delta \gamma} \bar{g}_{\mu \nu}\right)=0 \quad \forall \delta \gamma^{\mu} \in \Gamma\left(T M_{3}\right) \text { and } \forall \delta g_{\mu \nu} \in \operatorname{Sym}_{2}\left(T M_{3}\right) .
$$

This implies

$$
\mathcal{L}_{\bar{\gamma}} \psi^{\mu \nu}=\bar{D}^{\mu} \psi_{\mu \nu}=0
$$

In other words

$$
\operatorname{coker} Q_{0}=H_{1}(Q)=\operatorname{coker} \varphi
$$

The exactness of the sequence (7.12) implies therefore

$$
T_{\{\bar{g}, \bar{\gamma}\}} \mathcal{M} \simeq H_{0}(Q)=\frac{H_{-1}(Q) \oplus H_{1}(Q)}{\operatorname{Sym}_{2}^{\text {inv }}\left(T M_{3}\right)} \simeq \operatorname{ker} \varphi,
$$

where $T_{(\bar{\gamma}, \bar{g})} \mathcal{M}$ is the tangent to the space of physical moduli of the theory at a point $\left\{\bar{g}_{\mu \nu}, \bar{\gamma}^{\mu}\right\}$.

Hence $\bar{g}_{\mu \nu}$-isometries which commute with $\bar{\gamma}^{\mu}$ are in one-to-one correspondence with non-trivial deformations of a given Seifert structure $\left\{\bar{g}_{\mu \nu}, \bar{\gamma}^{\mu}\right\} \cdot \bar{\gamma}^{\mu}$ itself, of course, is always one of such isometries. The corresponding deformation is a rescaling of $\bar{\gamma}^{\mu}$. Since a rescaling of $\bar{\gamma}^{\mu}$ in the YM + CS action (3.27) can be reasorbed in a rescaling of the field $\tilde{A}$, the YM + CS partition function does not depend on this kind of deformation. In conclusion the parameter space, which the YM + CS partition function on a Seifert manifold depends on, is the quotient space

$$
\operatorname{ker} \varphi / \sim
$$

where the equivalence relation is

$$
\gamma^{\prime} \sim \gamma+\alpha \bar{\gamma}, \quad \gamma, \gamma^{\prime} \in \operatorname{ker} \varphi,
$$

with $\alpha$ constant. 


\section{The topological anomaly for the squashed spheres}

Let us now consider the squashed metric on $S_{3}:{ }^{10}$

$$
d s^{2}=\bar{g}_{\mu \nu}(x ; l, \tilde{l}) d x^{\mu} \otimes d x^{\nu}=\left(l^{2} \sin ^{2} \theta+\tilde{l}^{2} \cos ^{2} \theta\right) d \theta^{2}+l^{2} \cos ^{2} \theta d \phi_{1}^{2}+\tilde{l}^{2} \sin ^{2} \theta d \phi_{2}^{2}
$$

where $\phi_{1,2} \in[0,2 \pi]$ and $0 \leq \theta \leq \frac{\pi}{2}$, are the Hopf coordinates on $S_{3}$.

The vector field

$$
\bar{\gamma}^{\mu} \partial_{\mu}=\frac{1}{l} \partial_{\phi_{1}}+\frac{1}{\tilde{l}} \partial_{\phi_{2}}=\frac{\partial}{\partial y}
$$

is, for each value of the squashing parameters $(l, \tilde{l})$, an isometry of $\bar{g}_{\mu \nu}(x ; l, \tilde{l})$. A system of coordinates $(y, \alpha, \beta)$ adapted to the Seifert structure corresponding to $(l, \tilde{l})$ is defined by the relations

$$
\theta=\frac{\alpha}{2}, \quad \phi_{1}=\frac{y}{l}+\frac{\beta}{2 l}+\frac{\epsilon(\alpha, \beta)}{l}, \quad \phi_{2}=\frac{y}{\tilde{l}}-\frac{\beta}{2 \tilde{l}}+\frac{\epsilon(\alpha, \beta)}{\tilde{l}},
$$

and their inverse

$$
y=\frac{l \phi_{1}+\tilde{l} \phi_{2}}{2}-\epsilon(\alpha, \beta), \quad \beta=l \phi_{1}-\tilde{l} \phi_{2}, \quad \alpha=2 \theta .
$$

$\epsilon(\alpha, \beta)$ is an arbitrary local function which corresponds to abelian gauge transformations along the fiber of the fibration. The squashed metric (8.1) writes in these adapted coordinates as follows

$$
d s^{2}=(d y+a)^{2}+\frac{1}{4}\left[\frac{1}{2}\left(l^{2}+\tilde{l}^{2}+\left(\tilde{l}^{2}-l^{2}\right) \cos \alpha\right) d \alpha^{2}+\sin ^{2} \alpha d \beta^{2}\right]
$$

where the abelian gauge connection $a$ and its field strength $f^{(2)}$ are given by

$$
\begin{aligned}
a & =\frac{1}{2} \cos \alpha d \beta+d \epsilon, \\
f^{(2)} & =d a=-\frac{1}{2} \sin \alpha d \alpha d \beta .
\end{aligned}
$$

The curvature $R_{2}$ of the 2-dimensional metric $g_{i j}$ on the $S_{2}$ base of the Seifert fibration is

$$
\begin{aligned}
& R_{2}=\frac{8}{l^{2}} \frac{\left(b^{4}-1\right) \cos \alpha+2\left(b^{4}+1\right)}{\left(\left(b^{4}-1\right) \cos \alpha+b^{4}+1\right)^{2}}, \\
& \sqrt{g}=l \frac{1}{4} \sin \alpha \sqrt{\frac{b^{4}-1}{2} \cos \alpha+\frac{b^{4}+1}{2}},
\end{aligned}
$$

where we introduced the ratio

$$
b^{2} \equiv \frac{\tilde{l}}{l}
$$

\footnotetext{
${ }^{10}$ Our definitions and conventions for the Hopf coordinates for the squashed sphere are reviewed in appendix A.
} 
The scalar field which is dual to the abelian field strength is therefore

$$
f=\frac{\epsilon^{i j} \partial_{i} a_{j}}{\sqrt{g}}=\frac{2 \sqrt{2}}{l \sqrt{\left(b^{4}-1\right) \cos \alpha+b^{4}+1}}, \quad \sqrt{g} f=\frac{1}{2} \sin \alpha .
$$

We learnt in the previous section that, given $\bar{g}_{\mu \nu}(x ; l, \tilde{l})$ and $\bar{\gamma}=\frac{1}{l} \partial_{\phi_{1}}+\frac{1}{\tilde{l}} \partial_{\phi_{2}}$, the deformations of the Seifert structure are associated to the isometries which commute with $\bar{\gamma}$ modulo $\bar{\gamma}$. For generic $(l, \tilde{l})$ the isometries which commute with $\bar{\gamma}$ are $\partial_{\phi_{1}}$ and $\partial_{\phi_{2}}$. Hence we see that $b^{2}$ parametrizes precisely the inequivalent deformations of the Seifert structure around a generic point $b \neq 1$. The point $b=1$ corresponds to the "round" sphere, which has an enhanced symmetry $\mathrm{SU}(2)_{L} \times \mathrm{SU}(2)_{R}$. Around this point more general deformations are possible, since the isometries which commute with, let us say, $J_{3}^{R}$ form the full $\mathrm{SU}(2)_{L}$.

Let us compute the topological anomaly for the squashed sphere metric $\bar{g}_{\mu \nu}(x ; l, \tilde{l})$. Since $A_{1}^{(3)}\left[g_{\mu \nu}, \psi_{\mu \nu}\right]$ depends only on the conformal class of the metric, we can take, without loss of generality

$$
l=1, \quad \tilde{l}=b^{2}
$$

and put $\bar{g}_{\mu \nu}\left(x ; 1, b^{2}\right) \equiv \bar{g}_{\mu \nu}(x ; b)$. Then

$$
\bar{\psi}_{\mu \nu}(x ; b)=b \partial_{b} \bar{g}_{\mu \nu}(x ; b)=\left(\begin{array}{cccc}
4 b^{4} \cos ^{2} \theta & 0 & 0 \\
0 & 0 & 0 \\
0 & 0 & 4 b^{4} \sin ^{2} \theta
\end{array}\right)
$$

where the $b$-derivative is taken by keeping the Hopf coordinates constant. It is easy to verify that the topological anomaly for these backgrounds vanishes for all $b$ 's:

$$
A_{1}^{(3)}[\bar{g}, \bar{\psi}]=0 .
$$

This implies that the effective action $\Gamma\left[g_{\mu \nu}\right]$ evalutated for the squashed sphere metric $\bar{g}_{\mu \nu}(x ; b)$ is independent of $b$ :

$$
b \partial_{b} \Gamma\left[\bar{g}_{\mu \nu}(x ; b)\right]=b \partial_{b} \Gamma^{\text {Seif }}\left[\bar{g}_{i j}(X ; b), \bar{\sigma}(X ; b), \bar{a}_{i}(X ; b)\right]=0,
$$

where $X(x ; b) \equiv(y, \alpha, \beta)$ are the coordinates adapted to the Seifert structure parametrized by $b^{2}$.

However, we explained in section 6 that $\Gamma\left[g_{\mu \nu}\right]$ is not the action renormalized with the correct Seifert prescrition (6.15). The action $\tilde{\Gamma}^{\text {Seif }}\left[g_{i j}, \sigma, a_{i}\right]$ renormalized according to the Seifert prescription is given by (6.25). When this latter action is evaluated on $\bar{g}_{\mu \nu}(x ; b)$, one obtains

$$
\tilde{\Gamma}^{\text {Seif }}\left[\bar{g}_{i j}, \bar{\sigma}, \bar{a}_{i}\right]=\Gamma^{\text {Seif }}\left[\bar{g}_{i j}(X ; b), \bar{\sigma}(X ; b), \bar{a}_{i}(X ; b)\right]-\frac{c}{6} \Gamma_{\mathrm{WZ}}^{\text {Seif }}\left[\bar{g}_{i j}(X ; b), \bar{\sigma}(X ; b), \bar{a}_{i}(X ; b)\right] .
$$

We have just shown that, due to (8.12), the first (non-local) term in the r.h.s. of the equation above is $b$-independent. But the second (local) one is not. Indeed, by plugging (8.7) 
inside (6.23) one computes

$$
\begin{aligned}
\Gamma_{\mathrm{WZ}}^{\mathrm{Seif}}\left[\bar{g}_{i j}(X ; b), \bar{\sigma}(X ; b), \bar{a}_{i}(X ; b)\right] & =\int \frac{1}{2} \sqrt{g} f\left(f^{2}-R_{2}\right) \\
& =-(2 \pi)^{2}\left(b^{2}+\frac{1}{b^{2}}\right)
\end{aligned}
$$

which therefore encodes the whole dependence of the Seifert partition function on $b^{2}$ :

$$
Z^{\text {squashed }}(b)=\mathrm{e}^{i \tilde{\Gamma}^{\text {Seif }}\left[\bar{g}_{i j}, \bar{\sigma}, \bar{a}_{i}\right]}=\mathrm{e}^{i \frac{4 \pi^{2} c}{3}\left[\frac{1}{2}\left(b^{2}+\frac{1}{b^{2}}\right)-1\right]} Z^{\text {squashed }}(1),
$$

where $Z^{\text {squashed }}(1)$ is the partition function on the "round" sphere, corresponding to $b=1$.

Let us compare the anomaly equation (8.16) with the YM+CS partition function on the squashed sphere computed directly by means of localization in [32], taking for simplicity the case of $\mathrm{SU}(2)$ gauge group

$$
Z^{\text {squashed }}(b)=\int_{-\infty}^{\infty} \frac{d x}{2 \pi i} \mathrm{e}^{-\frac{i k x^{2}}{8 \pi}} \sinh \frac{i x b}{2} \sinh \frac{i x}{2 b} .
$$

Here the exponential in the integrand comes from the value of the action on the saddle point, while the hyperbolic sine factors are the results of the 1-loop determinants. Expressing the sinh factors in terms of exponentials, the integration reduces to the sum of Gaussian integrals:

$$
Z^{\text {squashed }}(b)=\mathrm{e}^{\frac{i \pi}{2 k}\left(b^{2}+\frac{1}{b^{2}}\right)-\frac{i \pi}{4}} Z_{\mathrm{CS}}^{\mathrm{CFT}}
$$

where

$$
Z_{\mathrm{CS}}^{\mathrm{CFT}}=\sqrt{\frac{2}{k}} \sin \frac{\pi}{k}
$$

is the round sphere $\mathrm{SU}(2)$ CS partition function, obtained by means of surgery from the CFT modular transformations of the WZW 2d conformal model. The result (8.18) obtained by direct computation agrees with our prediction (8.16) obtained from the topological anomaly and the Wess-Zumino Seifert action, once we insert the topological anomaly coefficient for $\mathrm{SU}(2), c_{\mathrm{SU}(2)}=\frac{1}{4 \pi} \frac{3}{k}$.

\section{Conclusions}

The current paradigm for localization relies on spinorial global supercharges. Since the fate and properties of quantum global symmetries are best studied by introducing background fields coupled to currents, the same paradigm has lead to studying the coupling of "physical" supersymmetric theories to off-shell supergravity. In particular the search for globally supersymmetric models has been reduced to the study of generalized covariantly constant spinors.

In this paper we proposed an alternative route. Localization is naturally understood in terms of topological scalar supercharges - i.e. in terms of topological theories and BRST symmetries. In this framework it is the coupling of topological field theories to topological 
gravity, not supergravity, which is relevant. For this reason we worked out the coupling of both CS and topological Yang Mills theory - i.e. of a generic vector twisted supermultiplet - to topological gravity. The BRST structure of the Chern-Simons supermultiplet looks very different from that of the topological YM theory, when the latter is presented in its familiar formulation valid in arbitrary dimension. We exhibited, however, a new formulation of the BRST transformations of topological YM in 3d purely in terms of the CS supermultiplet. This allowed us to derive a unique (anomalous) Ward identity which characterizes the coupling of a $3 \mathrm{~d}$ generic twisted vector supermultiplet to topological gravity.

One first advantage of the topological gravity viewpoint is that the structure of topological gravity is the same in all dimensions, a fact which makes the characterization of supersymmetric bosonic backgrounds straightforward. For example, in the context of $3 \mathrm{~d}$ gauge theories which is the one of this paper, we have seen that the Seifert condition emerges quite immediately without the need to go through generalized covariantly constant spinors or similar indirect routes peculiar to other approaches. Moreover we have found that, in the $3 \mathrm{~d}$ context, the off-shell coupling of topological (YM+CS) gauge theories to topological gravity is easily achieved by suitably covariantizing the "rigid" coboundary BRST operator with a universal term which is the form-contraction with the super-ghost field of topological gravity. We also discovered that the anti-fields of the BV formulations of CS theory are nothing but the auxiliary fields which are required to close off-shell the topological supersymmetry algebra.

But the real payoff of the topological approach was that it made straightforward to identify the subset of local topological transformations which preserves the Seifert backgrounds. These turned out to be $2 \mathrm{~d}$ topological gravity transformations coupled to topological abelian gauge transformations. This allowed us to give a cohomological characterization of the Seifert background moduli. Moreover we were able to explicitly solve the anomalous Ward identity associated to topological transformations of the gravitational background. The solution involved a Wess-Zumino local action, invariant under the reparametrizations which preserve the Seifert structure.

The triviality of the topological (framing) 3d anomaly when restricted on Seifert backgrounds shows, rigorously and in a completely regularization independent way, that the quantum effective action of the gauge theory on Seifert manifold depends on the Seifert moduli but not on the specific metric adapted to the Seifert structure which one picks to quantize the theory. The Wess-Zumino Seifert action also completely determines the dependence of the partition function on the Seifert moduli. We explicitly showed this in the case of the squashed sphere, for which we recovered the dependence of the partition function on the squashing modulus without computing any functional determinant.

Our discussion in this paper was restricted to (twisted) vector supermultiplets in $3 \mathrm{~d}$. It would be interesting to extend our results to (twisted) chiral matter. To do this it would be necessary to work out the coupling of topological chiral matter to topological gravitational backgrounds: something which, to our knowledge, has not be done yet. ${ }^{11}$ The dependence on the Seifert moduli of the quantum effective action of chiral theories is considerably more

\footnotetext{
${ }^{11}$ The BRST structure of rigid topological chiral matter in 3d has been described in [36].
} 
complicated than that of vector supermultiplets. We expect therefore that the coupling of topological chiral theories to topological gravitational backgrounds involves some new ingredients. It would be equally interesting to apply our methods to higher dimensions. Realizing this program might reduce the computation of the dependence on the moduli of quantum effective actions of localizable theories to the solution of appropriate anomalous Ward identities. We hope to come back to these problems in the future.

\section{Acknowledgments}

D.R. would like to thank B. Assel, S.J. Rey, A. Tomasiello, F. Yagi and A. Zaffaroni for interesting discussions. This work was supported in part by INFN, by Genoa University Research Project (P.R.A.) 2014 and by the National Research Foundation of Korea grants 2005-0093843, 2010-220-C00003 and 2012K2A1A9055280.

\section{A Squashed 3-spheres in the Hopf coordinates}

Let us start from the beginning and construct the squashed metric from $C^{2}$

$$
d s^{2}=\left|d z_{1}\right|^{2}+\left|d z_{2}\right|^{2}
$$

where

$$
z_{1}=\rho_{1} \mathrm{e}^{i \phi_{1}} \quad z_{2}=\rho_{2} \mathrm{e}^{i \phi_{2}}
$$

The $S_{3}$ is obtained by embedding the hypersurface

$$
\rho^{2} \equiv \frac{\rho_{1}^{2}}{l^{2}}+\frac{\rho_{2}^{2}}{\tilde{l}^{2}}=1
$$

in $C^{2}$, which gives

$$
\rho_{1}=l \cos \theta \quad \rho_{2}=\tilde{l} \sin \theta \quad 0 \leq \theta \leq \frac{\pi}{2}
$$

Hence

$$
\begin{aligned}
d s^{2} & =\sum_{i=1,2}\left|d \rho_{i}+i \rho_{i} d \phi_{i}\right|^{2}=d \rho_{1}^{2}+d \rho_{2}^{2}+\rho_{1}^{2} d \phi_{1}^{2}+\rho_{2}^{2} d \phi_{2}^{2} \\
& =\left(l^{2} \sin ^{2} \theta+\tilde{l}^{2} \cos ^{2} \theta\right) d \theta^{2}+l^{2} \cos ^{2} \theta d \phi_{1}^{2}+\tilde{l}^{2} \sin ^{2} \theta d \phi_{2}^{2}
\end{aligned}
$$

and the Killing vector which gives the Seifert structure is

$$
\bar{\gamma}^{\mu} \partial_{\mu}=\frac{1}{l} \partial_{\phi_{1}}+\frac{1}{\tilde{l}} \partial_{\phi_{2}}
$$




\section{B Gravitational CS action and the Seifert WZ action}

The topological anomaly is, by definition, non-trivial: there is no local functional of the 3 -dimensional metric, trasforming as a 3 -form, whose BRST variation gives the anomaly. As a matter of fact, one has

$$
A_{1}^{(3)}=s \Gamma_{\mathrm{GCS}}^{(3)}(g)
$$

where $\Gamma_{\mathrm{GCS}}^{(3)}$ is the 3-dimensional gravitational Chern-Simons action

$$
\Gamma_{\mathrm{GCS}}^{(3)}(g)=\operatorname{Tr}\left(\frac{1}{2} \Gamma d \Gamma+\frac{1}{3} \Gamma^{3}\right)
$$

Since $\Gamma_{\mathrm{GCS}}^{(3)}(g)$ is not a globally defined 3 -form on $M_{3}, A_{1}^{(3)}$ is indeed non-trivial.

Let us discuss the relation between the Seifert Wess-Zumino action (6.23) and the gravitational CS action (1.5). Let us introduce the 3-form

$$
\Gamma_{\mathrm{WZ}}^{(3) \text { Seif }}=\Gamma_{\mathrm{WZ}}^{\mathrm{Seif}} d y \frac{1}{2} \epsilon_{i j} d x^{i} d x^{j}
$$

Then of course

$$
s \int_{M_{3}}\left(\Gamma_{\mathrm{WZ}}^{(3) \text { Seif }}-\Gamma_{\mathrm{GCS}}^{(3)}\left[g_{i j}, \sigma, a_{i}\right]\right)=0
$$

where

$$
\left.\Gamma_{\mathrm{GCS}}^{(3)}\left[g_{i j}, \sigma, a_{i}\right] \equiv \Gamma_{\mathrm{GCS}}^{(3)}[g]\right|_{\mathcal{L}_{\bar{\gamma}} g}
$$

is the gravitational CS action evaluated for the metric adapted to the Seifert structure (6.10).

Therefore, there exists a local 2-form $\Omega^{(2)}$ such that

$$
\Gamma_{\mathrm{WZ}}^{(3) \text { Seif }}-\Gamma_{\mathrm{GCS}}^{(3)}\left[g_{i j}, \sigma, a_{i}\right]=d \Omega^{(2)}
$$

It turns out that

$$
\Omega^{(2)}=\frac{1}{4} \sqrt{g} f \epsilon_{i j} g^{j k} \Gamma_{l k}^{i} d x^{l} d y
$$

$\Gamma_{\text {GCS }}^{(3)}$ is not a globally defined 3-form: This fact is expressed by

$$
S_{\text {diff }} \Gamma_{\mathrm{GCS}}^{(3)}=d Q_{1}^{(2)}
$$

where $S_{\text {diff }}$ is the BRST operator associated to 3-dimensional diffeomorphisms:

$$
S_{\text {diff }}=\mathcal{L}_{\xi}-\left\{i_{\xi}, d\right\}
$$

with $\xi=\xi^{\mu} \partial_{\mu}$ the reparametrization ghost in 3-dimensions. $Q_{1}^{(2)}$ is the reparametrization anomaly in 2 dimensions:

$$
Q_{1}^{(2)}=\frac{1}{2} \operatorname{Tr} \mathrm{M} d \Gamma=\frac{1}{2} \partial_{\mu} \xi^{\nu} d \Gamma_{\nu}^{\mu}
$$


$\Gamma_{\mathrm{WZ}}^{(3) \text { Seif }}$ also is not invariant under $S_{\text {diff. }}$. However we can consider reparametrization ghosts $\xi^{\mu}$ which are invariant under the Reeb vector $\bar{\gamma}^{\mu}$. Let us denote by $S_{\text {diff }}^{\text {Seif }}$ the reparametrization BRST operator associated to such invariant reparametrization ghosts. The symmetry associated to $S_{\text {diff }}^{\text {Seif }}$ is the one corresponding to 2-dimensional diffeomorphisms and abelian gauge transformations.

Although $\Gamma_{\mathrm{GCS}}^{(3)}\left[g_{i j}, \sigma, a_{i}\right]$ is not invariant even under the restricted $\bar{S}_{\mathrm{diff}}, \Gamma_{\mathrm{WZ}}^{(3) \text { Seif }}$ is invariant:

$$
S_{\text {diff }}^{\text {Seif }} \Gamma_{\mathrm{WZ}}^{(3) \text { Seif }}=0
$$

as it is manifest from (6.23). This equations expresses the fact that $\Gamma_{\mathrm{WZ}}^{(3) \text { Seif }}$ is a form under reparametrizations which do not change the Seifert structure. Hence

$$
S_{\text {diff }}^{\text {Seif }}\left(\Gamma_{\mathrm{GCS}}^{(3)}\left[g_{i j}, \sigma, a_{i}\right]+d \Omega^{(2)}\right)=d\left(Q_{1}^{(2)}+S_{\text {diff }}^{\text {Seif }} \Omega^{(2)}\right)
$$

We conclude that

$$
Q_{1}^{(2)}=S_{\text {diff }}^{\text {Seif }} \Omega^{(2)}+d \Omega_{1}^{(1)}
$$

In other words the functional $\Omega^{(2)}$ trivializes the 2-dimensional gravitational anomaly: this is possibile since $\Omega^{(2)}$ is a functional not only of the 2-dimensional metric $g_{i j}$ but also of the abelian field strength $f$.

\section{Chern-Simons supergravity actions}

The bosonic fields of $3 \mathrm{~d} N=2$ supergravity include, beyond the metric $g_{\mu \nu}$, two gauge fields $A_{\mu}, V_{\mu}$ and a scalar $H$. Let us denote by $\Gamma\left[g_{\mu \nu}, A_{\mu}, V_{\mu}, H\right]$ the (non-local) effective action renormalized according to a prescription which preserves full reparametrizations and gauge invariance. ${ }^{12}$ For matter theories which involve only vector multiplets, the dependence on the supergravity bosonic backgrounds can be removed by subtracting from $\Gamma\left[g_{\mu \nu}, A_{\mu}, V_{\mu}, H\right]$ a local Chern-Simons supergravity action

$$
\begin{array}{r}
\Gamma_{\text {c.t. }}\left[g_{\mu \nu}, A_{\mu}, V_{\mu}, H\right]=\frac{1}{2} \int_{M_{3}}\left[\operatorname{Tr}\left(\omega d \omega+\frac{2}{3} \omega^{3}\right)+4\left(A-\frac{3}{2} V\right) d\left(A-\frac{3}{2} V\right)\right. \\
+(\text { fermions })]
\end{array}
$$

where $\omega$ is the gravitational spin-connection. The resulting effective action

$$
\tilde{\Gamma}=\Gamma\left[g_{\mu \nu}, A_{\mu}, V_{\mu}, H\right]-\Gamma_{\text {c.t. }}\left[g_{\mu \nu}, A_{\mu}, V_{\mu}, H\right]
$$

does not depend on $g_{\mu \nu}, A_{\mu}, V_{\mu}, H$ but it is not invariant under either "large" reparametrizations or "large" gauge transformations.

\footnotetext{
${ }^{12} \mathrm{By}$ "full" invariance we mean invariance under both "small" and "large" gauge and coordinate transformations.
} 
The bosonic supergravity backgrounds corresponding to the supersymmetric squashed sphere are

$$
\begin{aligned}
\bar{A} & =-\frac{1}{2}\left(1-\frac{1}{b f}\right) d \phi_{1}+\frac{1}{2}\left(1-\frac{b}{f}\right) d \phi_{2} \quad \bar{V}=0 \\
f^{2} & \equiv\left(b^{-2} \sin ^{2} \theta+b^{2} \cos ^{2} \theta\right) \\
\bar{H} & =-\frac{i}{f}
\end{aligned}
$$

The action (C.1) when evalutated for these backgrounds gives

$$
\Gamma_{\text {c.t. }}\left[\bar{g}_{\mu \nu}, \bar{A}_{\mu}, \bar{V}_{\mu}, \bar{H}\right]=2 \pi^{2}\left(b-\frac{1}{b}\right)^{2}
$$

This non-trivial $b$ dependence comes entirely from the gauge part of the Chern-Simons supergravity action (C.1): the gravitational part of (C.1) gives a $b$-independent contribution.

Since $\tilde{\Gamma}$ is independent of the bosonic backgrounds, its value for the squashed sphere does not depend on $b$. Hence the (non-local) reparametrization invariant and gauge invariant effective action $\Gamma\left[g_{\mu \nu}, A_{\mu}, V_{\mu}, H\right]$ evaluated for the backgrounds (C.3) gives

$$
\Gamma\left[\bar{g}_{\mu \nu}, \bar{A}_{\mu}, \bar{V}_{\mu}, \bar{H}\right]=\Gamma_{\text {c.t. }}\left[\bar{g}_{\mu \nu}, \bar{A}_{\mu}, \bar{V}_{\mu}, \bar{H}\right]=2 \pi^{2}\left(b-\frac{1}{b}\right)^{2}
$$

up to a $b$-independent constant. However, as we explained in section (6), neither $\Gamma\left[g_{\mu \nu}, A_{\mu}, V_{\mu}, H\right]$ nor $\tilde{\Gamma}$ is the effective action renormalized with the prescription which is appropriate for quantization on Seifert manifolds. The Seifert quantum action is obtained from $\Gamma\left[g_{\mu \nu}, A_{\mu}, V_{\mu}, H\right]$ by subtracting local counterterms which preserve invariance under reparametrizations and gauge transformations which are compatible with the Seifert structure and, at the same time, restore the symmetry under topological transformations of metric and gauge fields which do not change the Seifert moduli.

In section 6 we showed that the appropriate local gravitational counterterm is proportional to the Seifert Wess-Zumino action (6.23). In the supergravity framework we have to add to this the analogous gauge local counterterm, to obtain ${ }^{13}$

$$
\Gamma_{\mathrm{c.t.}}^{\mathrm{Seif}}=\Gamma_{\mathrm{WZ}}^{\mathrm{Seif}}\left[g_{i j}, \sigma, a_{i}\right]+4 \Gamma_{\text {gauge }}^{\mathrm{Seif}}\left[\phi, A^{\prime}\right]
$$

where

$$
\Gamma_{\text {gauge }}^{\text {Seif }}\left[\phi, A^{\prime}\right]=\int\left(\frac{1}{2} k d k \phi^{2}+k F^{\prime} \phi\right)
$$

In these formulas, $k$ is the contact form associated to the Seifert structure

$$
k(\bar{\gamma})=1
$$

$\bar{\gamma}$ is the Reeb Killing vector field and

$$
\phi=i_{\bar{\gamma}}(A) \quad A=k \phi+A^{\prime} \quad F^{\prime}=d A^{\prime} \quad \mathcal{L}_{\bar{\gamma}} A^{\prime}=i_{\bar{\gamma}}\left(A^{\prime}\right)=0
$$

is a gauge-background compatible with the Seifert structure.

\footnotetext{
${ }^{13}$ The factor 4 in front of the gauge counterterm is dictated by the presence of the same factor in (C.1).
} 
$\Gamma_{\text {gauge }}^{\text {Seif }}\left[\phi, A^{\prime}\right]$ is invariant under reparametrizations which preserve the Seifert structure. Its topological variation reproduces the topological gauge anomaly encoded in the $3 \mathrm{~d}$ Abelian Chern-Simons action:

$$
\begin{array}{r}
s \Gamma_{\text {gauge }}^{\text {Seif }}\left[\phi, A^{\prime}\right]=\int\left[\chi k\left(d k \phi+F^{\prime}\right)+\psi^{\prime} d(k \phi)\right]=\int \psi F \\
s A=\psi=k \chi+\psi^{\prime} \quad s \phi=\chi \quad s A^{\prime}=\psi^{\prime}
\end{array}
$$

The important point is that $\Gamma_{\text {gauge }}^{\text {Seif }}\left[\phi, A^{\prime}\right]$ coincides with $3 \mathrm{~d}$ Chern-Simons action

$$
\Gamma_{\mathrm{CS}}[A]=\frac{1}{2} \int A d A
$$

when this is evalutated on gauge backgrounds of the Seifert form (C.9)

$$
\Gamma_{\mathrm{CS}}[A]=\Gamma_{\text {gauge }}^{\text {Seif }}\left[\phi, A^{\prime}\right]
$$

Therefore the Seifert gauge counterterms when evaluated for the squashed sphere backgrounds gives the very same function of $b$ (C.4) which is produced by the gauge counterterms in (C.1)

$$
4 \Gamma_{\text {gauge }}^{\text {Seif }}\left[\bar{\phi}, \bar{A}^{\prime}\right]=2 \pi^{2}\left(b-\frac{1}{b}\right)^{2}
$$

where $\bar{\phi}$ and $\bar{A}^{\prime}$ are the squashed sphere backgrounds

$$
\bar{\gamma}=b \partial_{\phi_{1}}+\frac{1}{b} \partial_{\phi_{2}} \quad \bar{\phi}=-\frac{1}{2}\left(b-\frac{1}{b}\right)
$$

This means that when one evaluates the effective action renormalized with the Seifert prescription

$$
\tilde{\Gamma}^{\text {Seif }}=\Gamma\left[g_{\mu \nu}, A_{\mu}, V_{\mu}, H\right]-\Gamma_{\text {c.t. }}^{\text {Seif }}\left[g_{\mu \nu}, A_{\mu}, V_{\mu}, H\right]
$$

on the squashed sphere backgrounds, the dependence on $b$ associated to the gauge counterterms drops out and one is left with the dependence on $b$ dictated by the gravitational Seifert Wess-Zumino action (6.23)

$$
\begin{aligned}
\tilde{\Gamma}^{\text {Seif }}\left[\bar{g}_{\mu \nu}, \bar{A}_{\mu}, \bar{V}_{\mu}, \bar{H}\right] & =\Gamma\left[\bar{g}_{\mu \nu}, \bar{A}_{\mu}, \bar{V}_{\mu}, \bar{H}\right]-\Gamma_{\text {c.t. }}^{\text {Seif }}\left[\bar{g}_{\mu \nu}, \bar{A}_{\mu}, \bar{V}_{\mu}, \bar{H}\right] \\
& =2 \pi^{2}\left(b-\frac{1}{b}\right)^{2}-\Gamma_{\mathrm{WZ}}^{\text {Seif }}\left[\bar{g}_{i j}, \bar{\sigma}, \bar{a}_{i}\right]-2 \pi^{2}\left(b-\frac{1}{b}\right)^{2} \\
& =-\Gamma_{\mathrm{WZ}}^{\text {Seif }}\left[\bar{g}_{i j}, \bar{\sigma}, \bar{a}_{i}\right]=-(2 \pi)^{2}\left(b^{2}+\frac{1}{b^{2}}\right)
\end{aligned}
$$

in agreement with the result we obtained in the topological framework.

Open Access. This article is distributed under the terms of the Creative Commons Attribution License (CC-BY 4.0), which permits any use, distribution and reproduction in any medium, provided the original author(s) and source are credited. 


\section{References}

[1] E. Witten, Mirror manifolds and topological field theory, hep-th/9112056 [INSPIRE].

[2] E. Witten, Two-dimensional gauge theories revisited, J. Geom. Phys. 9 (1992) 303 [hep-th/9204083] [INSPIRE].

[3] N.A. Nekrasov, Seiberg-Witten prepotential from instanton counting, Adv. Theor. Math. Phys. 7 (2004) 831 [hep-th/0206161] [INSPIRE].

[4] V. Pestun, Localization of gauge theory on a four-sphere and supersymmetric Wilson loops, Commun. Math. Phys. 313 (2012) 71 [arXiv:0712.2824] [InSPIRE].

[5] C. Closset, T.T. Dumitrescu, G. Festuccia and Z. Komargodski, Supersymmetric field theories on three-manifolds, JHEP 05 (2013) 017 [arXiv:1212.3388] [INSPIRE].

[6] L. Rozansky, A large $k$ asymptotics of Witten's invariant of Seifert manifolds, Commun. Math. Phys. 171 (1995) 279 [hep-th/9303099] [INSPIRE].

[7] M. Mariño, Chern-Simons theory, matrix integrals and perturbative three manifold invariants, Commun. Math. Phys. 253 (2004) 25 [hep-th/0207096] [INSPIRE].

[8] C. Beasley and E. Witten, Non-abelian localization for Chern-Simons theory, J. Diff. Geom. 70 (2005) 183 [hep-th/0503126] [INSPIRE].

[9] M. Blau and G. Thompson, Chern-Simons theory on S1-bundles: abelianisation and q-deformed Yang-Mills theory, JHEP 05 (2006) 003 [hep-th/0601068] [INSPIRE].

[10] A. Kapustin, B. Willett and I. Yaakov, Exact results for Wilson loops in superconformal Chern-Simons theories with matter, JHEP 03 (2010) 089 [arXiv:0909.4559] [INSPIRE].

[11] N. Hama, K. Hosomichi and S. Lee, SUSY gauge theories on squashed three-spheres, JHEP 05 (2011) 014 [arXiv:1102.4716] [INSPIRE].

[12] X. Huang, S.-J. Rey and Y. Zhou, Three-dimensional SCFT on conic space as hologram of charged topological black hole, JHEP 03 (2014) 127 [arXiv:1401.5421] [INSPIRE].

[13] E. Witten, Quantum field theory and the Jones polynomial, Commun. Math. Phys. 121 (1989) 351 [inSPIRE].

[14] G. Festuccia and N. Seiberg, Rigid supersymmetric theories in curved superspace, JHEP 06 (2011) 114 [arXiv:1105.0689] [INSPIRE].

[15] C. Closset, T.T. Dumitrescu, G. Festuccia, Z. Komargodski and N. Seiberg, Contact terms, unitarity and F-maximization in three-dimensional superconformal theories, JHEP 10 (2012) 053 [arXiv: 1205.4142] [INSPIRE].

[16] C. Closset, T.T. Dumitrescu, G. Festuccia, Z. Komargodski and N. Seiberg, Comments on Chern-Simons contact terms in three dimensions, JHEP 09 (2012) 091 [arXiv:1206.5218] [INSPIRE].

[17] A.S. Schwarz, The partition function of degenerate quadratic functional and Ray-Singer invariants, Lett. Math. Phys. 2 (1978) 247 [INSPIRE].

[18] J.F. Schonfeld, A mass term for three-dimensional gauge fields, Nucl. Phys. B 185 (1981) 157 [InSPIRE].

[19] R. Jackiw and S. Templeton, How superrenormalizable interactions cure their infrared divergences, Phys. Rev. D 23 (1981) 2291 [INSPIRE]. 
[20] S. Deser, R. Jackiw and S. Templeton, Three-dimensional massive gauge theories, Phys. Rev. Lett. 48 (1982) 975 [INSPIRE].

[21] S. Deser, R. Jackiw and S. Templeton, Topologically massive gauge theories, Annals Phys. 140 (1982) 372 [Erratum ibid. 185 (1988) 406] [Annals Phys. 281 (2000) 409] [INSPIRE].

[22] C. Imbimbo, The coupling of Chern-Simons theory to topological gravity, Nucl. Phys. B 825 (2010) 366 [arXiv:0905.4631] [INSPIRE].

[23] L. Baulieu and I.M. Singer, Topological Yang-Mills symmetry, Nucl. Phys. Proc. Suppl. 5B (1988) 12 [InSPIRE].

[24] S. Ouvry, R. Stora and P. van Baal, On the algebraic characterization of Witten's topological Yang-Mills theory, Phys. Lett. B 220 (1989) 159 [INSPIRE].

[25] L. Baulieu and I.M. Singer, The topological $\sigma$ model, Commun. Math. Phys. 125 (1989) 227 [INSPIRE].

[26] H. Kanno, Weyl algebra structure and geometrical meaning of BRST transformation in topological quantum field theory, Z. Phys. C 43 (1989) 477 [InSPIRE].

[27] S. Axelrod and I.M. Singer, Chern-Simons perturbation theory. 2, J. Diff. Geom. 39 (1994) 173 [hep-th/9304087] [INSPIRE].

[28] M. Alexandrov, M. Kontsevich, A. Schwartz and O. Zaboronsky, The geometry of the master equation and topological quantum field theory, Int. J. Mod. Phys. A 12 (1997) 1405 [hep-th/9502010] [INSPIRE].

[29] J.H. Schwarz, Superconformal Chern-Simons theories, JHEP 11 (2004) 078 [hep-th/0411077] [INSPIRE].

[30] C. Klare, A. Tomasiello and A. Zaffaroni, Supersymmetry on curved spaces and holography, JHEP 08 (2012) 061 [arXiv: 1205.1062] [INSPIRE].

[31] J. Kallen, Cohomological localization of Chern-Simons theory, JHEP 08 (2011) 008 [arXiv: 1104.5353] [INSPIRE].

[32] N. Hama, K. Hosomichi and S. Lee, Notes on SUSY gauge theories on three-sphere, JHEP 03 (2011) 127 [arXiv: 1012.3512] [INSPIRE].

[33] W.A. Bardeen and B. Zumino, Consistent and covariant anomalies in gauge and gravitational theories, Nucl. Phys. B 244 (1984) 421 [INSPIRE].

[34] C. Closset, T.T. Dumitrescu, G. Festuccia and Z. Komargodski, The geometry of supersymmetric partition functions, JHEP 01 (2014) 124 [arXiv:1309.5876] [INSPIRE].

[35] C. Closset, T.T. Dumitrescu, G. Festuccia and Z. Komargodski, From rigid supersymmetry to twisted holomorphic theories, Phys. Rev. D 90 (2014) 085006 [arXiv:1407.2598] [INSPIRE].

[36] K. Ohta and Y. Yoshida, Non-abelian localization for supersymmetric Yang-Mills-Chern-Simons theories on Seifert manifold, Phys. Rev. D 86 (2012) 105018 [arXiv: 1205.0046] [INSPIRE]. 